\title{
Impaired dopamine metabolism in Parkinson's disease pathogenesis
}

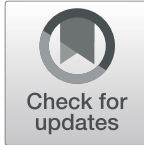

\author{
Anna Masato ${ }^{1}$ (D), Nicoletta Plotegher ${ }^{1}$ (D), Daniela Boassa ${ }^{2}$ (D) and Luigi Bubacco ${ }^{1 *}$ (D)
}

\begin{abstract}
A full understanding of Parkinson's Disease etiopathogenesis and of the causes of the preferential vulnerability of nigrostriatal dopaminergic neurons is still an unsolved puzzle. A multiple-hit hypothesis has been proposed, which may explain the convergence of familial, environmental and idiopathic forms of the disease. Among the various determinants of the degeneration of the neurons in Substantia Nigra pars compacta, in this review we will focus on the endotoxicity associated to dopamine dyshomeostasis. In particular, we will discuss the relevance of the reactive dopamine metabolite 3,4-dihydroxyphenylacetaldehyde (DOPAL) in the catechol-induced neurotoxicity. Indeed, the synergy between the catechol and the aldehyde moieties of DOPAL exacerbates its reactivity, resulting in modification of functional protein residues, protein aggregation, oxidative stress and cell death. Interestingly, aSynuclein, whose altered proteostasis is a recurrent element in Parkinson's Disease pathology, is considered a preferential target of DOPAL modification. DOPAL triggers aSynuclein oligomerization leading to synapse physiology impairment. Several factors can be responsible for DOPAL accumulation at the pre-synaptic terminals, i.e. dopamine leakage from synaptic vesicles, increased rate of dopamine conversion to DOPAL by upregulated monoamine oxidase and decreased DOPAL degradation by aldehyde dehydrogenases. Various studies report the decreased expression and activity of aldehyde dehydrogenases in parkinsonian brains, as well as genetic variants associated to increased risk in developing the pathology. Thus, we discuss how the deregulation of these enzymes might be considered a contributing element in the pathogenesis of Parkinson's Disease or a down-stream effect. Finally, we propose that a better understanding of the impaired dopamine metabolism in Parkinson's Disease would allow a more refined patients stratification and the design of more targeted and successful therapeutic strategies.
\end{abstract}

Keywords: Parkinson's disease, Selective vulnerability, Dopamine, DOPAL, aSynuclein, Aldehyde dehydrogenase

\section{Background}

Parkinson's Disease (PD) is an age-related, severe neurodegenerative movement disorder. The pathology affects about $1 \%$ of the population over 65 years old and more than $4-5 \%$ over 80 , being the latter the current average life expectancy in the European Community [1, 2]. Clinical PD is a multi-factorial pathology and most of the cases are classified as sporadic with an undefined aetiology, while only $5-10 \%$ of cases have genetic causes. At the histological level, the progressive neuronal loss corresponds to the accumulation of proteinaceous intracytoplasmic inclusions, named Lewy Bodies (LBs), in

\footnotetext{
* Correspondence: luigi.bubacco@unipd.it

${ }^{1}$ Department of Biology, University of Padova, Padova, Italy

Full list of author information is available at the end of the article
}

which amyloid fibrils of the presynaptic protein $\alpha$ Synuclein ( $\alpha$ Syn) are the main constituent [3].

It has been proposed that, during the development of the pathology, neurodegeneration gradually interests different regions of the brain although it mostly affects the nigrostriatal circuits in the midbrain. This results in the typical motor symptoms, as the nigrostriatal pathway is involved in voluntary movement coordination of the body. Indeed, after the loss of more than $80 \%$ of the dopaminergic neurons in the Substantia Nigra pars compacta (SNpc), parkinsonian syndrome manifests with tremor at rest, rigidity, slowness or absence of voluntary movement, postural instability and freezing $[4,5]$. This view however, is still object of debate, as it has been recently challenged by Engelender and Isacson, who argued that

(c) The Author(s). 2019 Open Access This article is distributed under the terms of the Creative Commons Attribution 4.0 International License (http://creativecommons.org/licenses/by/4.0/), which permits unrestricted use, distribution, and 
the observed ascending progression of the disease may result from a combination of a diverse vulnerability of Central Nervous System and Peripheral Nervous System, as well as different "functional reserve" of the neurons involved [6].

The identification of causative factors responsible for the preferential vulnerability of dopaminergic neurons of $\mathrm{SNpc}$ is still an unsolved quest in PD research and its purported molecular determinants have been recently reviewed by Brichta and Greengard [7]. The remaining challenge is still in understanding why mutations in various proteins with different or unclear physiological functions converge to similar pathological phenotypes, which are also observed in idiopathic PD cases [8]. Conversely, familial, environmental and idiopathic PD forms present some differences from both the histopathological and clinical point of view. For example, PD patients carrying Parkin, Pink1 or Lrrk2 mutation do not always present LBs $[8,9]$. Moreover, patients differ in terms of age of onset, disease severity, progression of the neurodegeneration and type of symptoms (motor and non-motor).

On this ground, a multiple-hit hypothesis for PD pathogenesis has been put forward [10, 11]. According to this hypothesis, several risk factors, both genetic and environmental, concomitantly affect neuronal homeostasis resulting in progressive neurodegeneration [10, 11]. This hypothesis may explain both similarities and divergences in the different PD forms and it would allow patient stratification. As Surmeier and colleagues recently reviewed, the analysis of morphological, functional and molecular peculiarities of the SNpc dopaminergic neurons is starting to shed some light on their selective vulnerability in $\mathrm{PD}[5,12]$. As main features, this neuronal population presents an intrinsic low calcium buffering capacity and the ability to perform pace-making activity [13]. Moreover, the dopaminergic neurons carry the machinery to metabolize and catabolize dopamine (DA), the neurotransmitter synthetized and secreted in the nigrostriatal pathway.

Among these important aspects (which may be not mutually exclusive in determining dopaminergic neurons vulnerability), our interest here will mainly focus on the role of DA metabolism and catabolism in PD etiopathogenesis. Indeed, the endotoxicity derived from increasing DA levels, DA oxidation and its reactive catabolites, is recognised as one of the major causes of oxidative stress in PD [14-17]. Interestingly, several PD-related proteins appeared to participate in the modulation of the dopaminergic pathway in health and disease [18, 19]. Therewithal, $\alpha$ Syn, whose altered proteostasis is primarly involved in molecular mechanisms responsible for neuronal death, has been highlighted as preferential target of DA-related neurotoxicity $[20,21]$.
In the last decades, the concept that a dyshomeostasis of catechol amines may lead to endotoxicity has been extended to DA catabolites, as many studies revealed impaired DA metabolites in PD models and autoptic samples [22]. Among the several metabolites monitored, attention was addressed on 3,4-dihydroxyphenylacetaldehyde (DOPAL), a toxic DA catabolite. In this review, we aim to discuss evidence that support DOPAL involvement in the pathogenesis of $\mathrm{PD}$, its potential synergy in $\alpha$ Syninduced pathology and whether DOPAL toxicity might contribute to rationalize the deleterious effects on nigral neurons that have been referred solely to DA.

\section{3,4-Dihydroxyphenylacetaldehyde: a relevant player in dopaminergic neuron degeneration}

DA levels within SNpc neurons are strictly regulated, as an equilibrium among synthesis, synaptic vesicle loading, uptake from the extracellular space and catabolic degradation [16]. As showed in Fig. 1, DA catabolism starts with the oxidative deamination, a reaction mediated by the mitochondrial monoamine oxidase (MAO), which also generates $\mathrm{H}_{2} \mathrm{O}_{2}$ and ammonia. The resulting product, DOPAL, is further metabolized either to 3,4-dihydroxyphenylacetic acid (DOPAC) or 3,4-dihydroxyphenylethanol (DOPET) by aldehyde dehydrogenase (ALDH) or by aldehyde/aldose reductase (ALR/AR), respectively.

Although DOPAL is a physiological intermediate in DA catabolism, it resulted to be an endogenous neurotoxin [23]. Being an aldehyde, DOPAL is a very electrophilic molecule, prone to induce covalent modification of nucleophile functional groups in the cytoplasmic milieu [24]. DOPAL concentration in $\mathrm{SNpc}$ dopaminergic neurons has been estimated to be around $2-3 \mu \mathrm{M}$, a level compatible with the affinity reported for the DOPAL detoxifying enzymes previously mentioned (0.4-1 $\mu \mathrm{M}$ for ALDHs) [24]. Concentrations higher than physiological $(>6 \mu \mathrm{M})$ have been described as a threshold for cytotoxic effects in various cell lines [24]. Thereafter, the work of Burke et al. in 2003 provided substantial evidence of DOPAL neurotoxicity in vivo [25]. DOPAL injection in rat nigral dopaminergic neurons resulted in detrimental neuronal loss, more pronounced than that induced by administration of DA or its metabolites (DOPAC, DOPET, HVA). More recently, a post mortem study on sporadic PD patients' brains revealed DOPAL build-up relative to DA in the putamen of PD subjects compared to healthy controls [26]. The levels of DA and its catabolites was determined by High Pressure Liquid Chromatographic separation coupled to Electro-Chemical Detection (HPLC-ECD) [27-29]. This technique, which is considered the 'gold standard' for catechols quantification in cells and tissues, allows singling out DOPAL from other catecholamines, based on its unique electro-chemical properties. Using the same technique, other correlated studies also reported decreased DOPAC:DOPAL ratio in PD, 


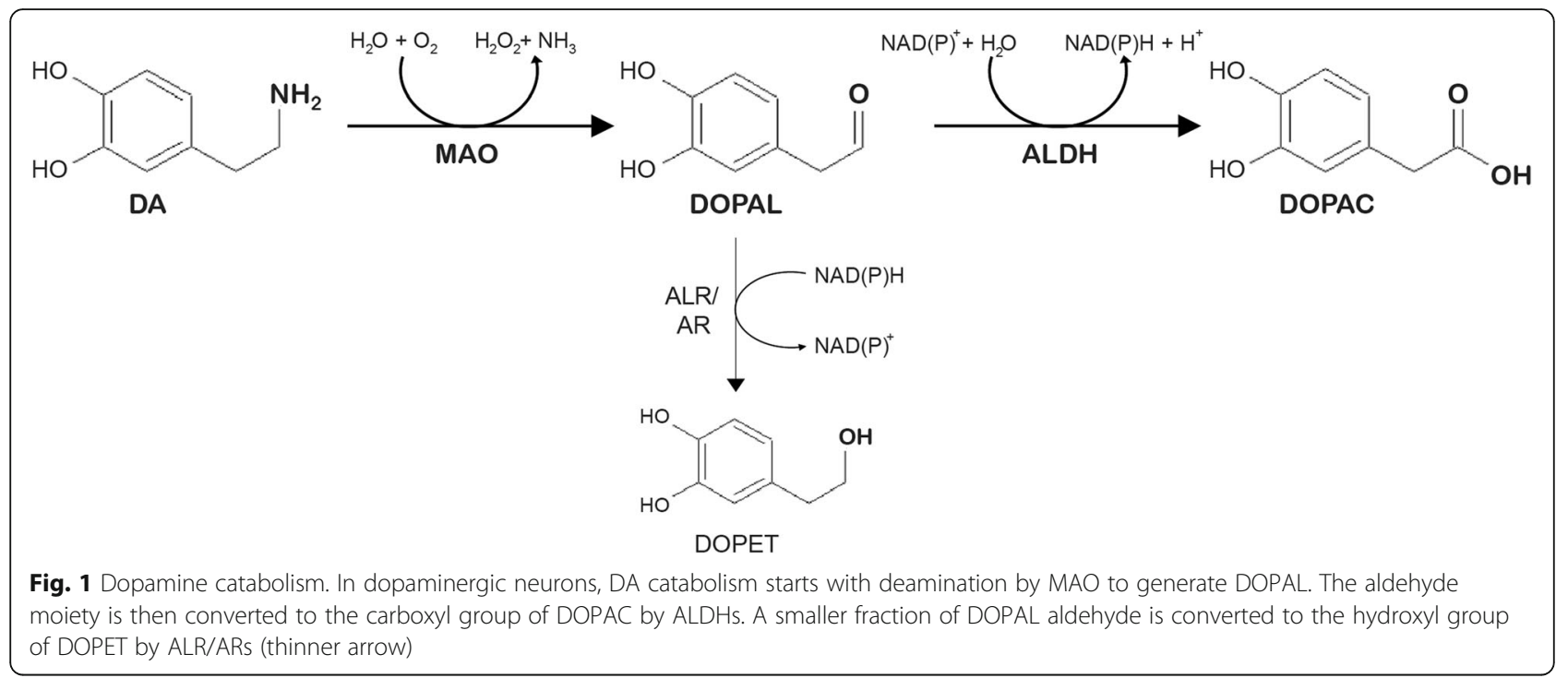

together with lowered vesicular sequestration of DA through the vesicular monoamine transporter type-2 (VMAT-2) [26, 27, 30]. Moreover, a decreased DOPAC content in cerebrospinal fluid (CSF) from samples of PD patients was measured, combined by 5-S-cysteinyl-DA/ DOPAC ratios averaged more than twice compared to controls [31]. In this frame, these seminal results prompted the formulation of the Catecholaldehyde hypothesis, which underscores the key role of DOPAL in the molecular mechanisms responsible for $\mathrm{SNpc}$ degeneration in PD [23, 25, 32-35].

DOPAL is a highly reactive molecule, which presents two functional groups that may account for its toxicity.
These are the aldehyde and catechol moieties, which can both contribute to DOPAL reactivity toward proteins (Fig. 2). The first one targets mainly primary amines and the second thiols [36]. Of interest, the two moieties do not act independently of each other, in fact the oxidation of the catechol ring enhances the Schiff base reaction between the aldehyde moiety of DOPAL and primary amines [37]. Also, the oxidation of the catechol is required for the addiction of thiols to the aromatic ring. This implies that DOPAL is prone to covalently modify amino acid residues i.e. lysines and cysteines.

As mentioned above, the HPLC-ECD is the most reliable method to quantify the concentrations of catechols

\section{a Schiff-base reaction with Lysines}<smiles>C[AsH2]NC(=O)CCc1ccc(O)c(O)c1</smiles>

\section{b Oxidation and reaction with Cysteines}<smiles>CSc1cc(CC=O)cc(O)c1O</smiles>

Fig. 2 DOPAL reactivity and reported neurotoxic molecular mechanisms. DOPAL reactivity is due to both the aldehyde and the catechol moiety, respectively resulting in covalent modification of primary amines and thiols (i.e. lysine and cysteine residues of proteins) [36-38]. a DOPAL addiction to lysines is the result of a Schiff-base reaction between the aldehyde and the primary amine of the lysine's lateral chain, with the release of a molecule of water. $\mathbf{b}$ In oxidative conditions, the catechol group has the tendency to auto-oxidation, with production of quinones and oxygen radical species [39]. Also, the oxidized cathecol is reactive towards the thiols of cysteines 
in solution. However, this method does not allow to measure the fraction of DOPAL that is bound to proteins. So far, the detection of catechol-modified proteins from cell lysates has been performed by SDS-Page followed by the staining with nitroblue tetrazolium (NBT), a redox-cycling dye for the detection of catechol adducts [34]. Alternatively, the protein pull-down assay with aminophenylboronic acid (APBA) resin allows the isolation of catechol-modified proteins from cell lysates [40, 41], with the caveat that it also binds glycosylated proteins. More recently, the near Infrared Fluorescence (nIRF) scanning was applied to the detection and quantification of o-quinones in cells and tissues, as well as proteins modified by oxidized catechols [18, 20, 42]. This method relies on the ability of quinones to emit a fluorescence signal after excitation at $700 \mathrm{~nm}$ upon stimulation at $685 \mathrm{~nm}$ [43]. Anyway, all these other techniques are way less sensitive than the HPLC-ECD, leaving a rigorous DOPAL quantification in biological samples a challenging task. As a consequence, the development of tools aimed to precisely quantify catechol levels and DOPAL-modified macromolecules in both experimental models and patients' samples is needed.

DOPAL reactivity and accumulation in cells are believed to be detrimental to neurons and possibly responsible of different neurotoxic mechanisms. These were ascribed to DOPAL alone or to DOPAL-modified molecules, as summarized in Fig. 3.
DOPAL-induced proteostasis alteration (Fig. 3a): effects on protein aggregation, on functional lysines and competition with other post-translational modifications The high reactivity of both functional groups of DOPAL results in protein cross-linking which leads to protein aggregation. This was demonstrated by several in vitro studies, in which DOPAL was incubated with Glyceraldehyde-3-phosphate-dehydrogenase (GAPDH) and Bovine Serum Albumin (BSA) as model proteins [34, 36]. More interestingly, DOPAL was shown to trigger $\alpha$ Syn oligomerization to generate SDS-resistant high molecular weight species to whom pathological relevance in PD has been attributed [38, 41, 42, 44]. This issue will be extensively discussed in the following paragraph "Linking the Catecholaldehyde Hypothesis to $\alpha$ Syn-induced pathology".

Lysine residues are often subjected to post-translational modifications (i.e. ubiquitination, SUMOylation, acetylation) that are important in regulating protein clearance, protein subcellular localization, protein-protein interactions and protein secretion through extra-cellular vesicles [45]. Of note, ubiquitin itself possesses seven functional lysines, through which poly-ubiquitin chains are synthetized to target proteins in different cellular compartments. It has been recently demonstrated that, in vitro, DOPAL modifies ubiquitin lysines and promotes ubiquitin oligomerization [42]. In the same work, DOPAL treatment on PC-12 cells resulted in accumulation of ubiquitinated proteins [42]. In this frame, more needs to be done to
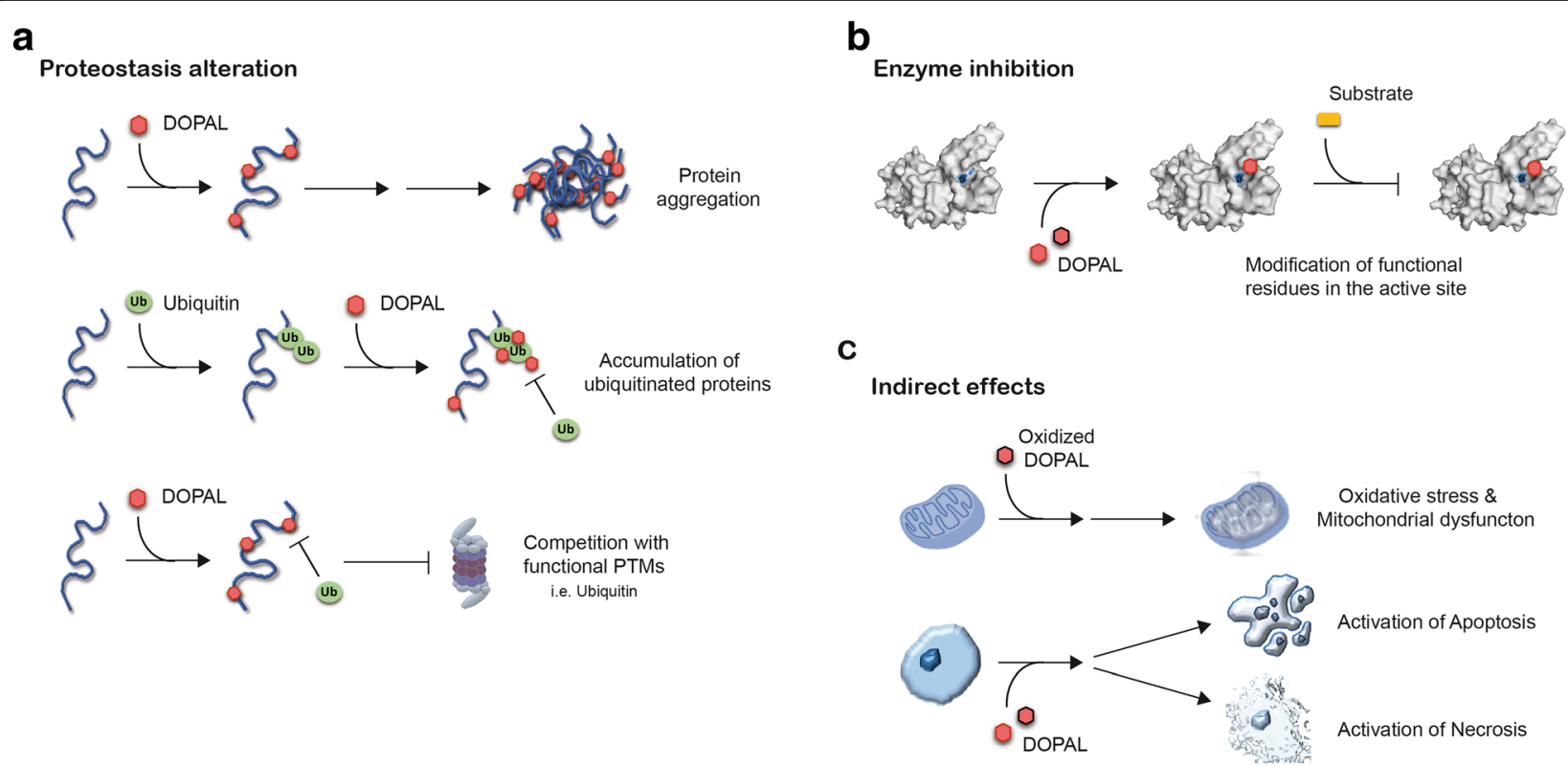

Fig. 3 DOPAL reported neurotoxic molecular mechanisms. DOPAL build-up in SNpc dopaminergic neurons triggers multiple neurotoxic mechanisms: a alteration of neuronal proteostasis, in terms of protein aggregation [34, 36, 38, 41, 44], competition with functional post-translational modifications (PTMs, i.e. ubiquitination, SUMOylation, acetylation) and accumulation of ubiquitinated proteins [42, 45]; b enzyme inhibition (PDB: 4i1f, in the figure) [46-48]; $\mathbf{c}$ indirect effects, which imply oxidative stress [39], mitochondrial dysfunction [17, 49-51], activation of necrotic and apoptotic pathways [23, 24, 33] 
explore a potential scenario in which the chemical modification by DOPAL of lysine-rich proteins in neurons, would affect not only their proper functions but also their capability to be tagged by ubiquitin. As a consequence, dysfunctional DOPAL-modified proteins that should be targeted for clearance would end up in an aberrant accumulation because of DOPAL competition for their ubiquitination sites or the impairment of the ubiquitination pathway itself.

Along the same line, not only ubiquitin and the ubiquitination pathway, but also small ubiquitin-like modifier (SUMO) proteins may be the targets for DOPAL modifications. Four SUMO paralogues have been identified in humans (SUMO-1 to SUMO-4) and similarly to ubiquitin they present a large number of lysines in their sequence (for example, 11 lysines out of 101 amino acids in hSUMO-1). SUMOs substrates, SUMOs conjugation machinery and the specificity of paralogues towards diverse proteins are still object of extensive research. However, it is known that SUMOylation can act on several cellular processes as transcription and protein localization, by regulating protein-protein interaction and substrate conformational changes [52]. For instance, in neurons, SUMOylation of the glutamate receptor subunit 6 is responsible for the endocytosis of the receptor at the plasma membrane [53]. Limited information about the role of SUMOs in PD is available, but it was shown that this pathway is crucial for cellular function and survival. In fact, when Ubc9, which is required for conjugation of SUMO proteins to their substrate, is depleted, cells present nuclear abnormalities and undergo apoptosis [54]. Coherently, SUMOylation was reported to mediate $\alpha$ Syn sorting into lumen of vesicles and attenuate $\alpha$ Syn aggregation and toxicity [55-57]. It is then feasible to reason that covalent modifications to SUMO lysines due to DOPAL accumulation may reduce the amount of SUMOs available for the tight regulation of essential cellular processes. At the same time, DOPAL-modified lysines on the substrate proteins would affect the possibility for them to be SUMOylated.

Lysines modification by DOPAL may also impact on another relevant pathway for the regulation of different cellular functions, i.e. lysines acetylation. This process relies on acetyl-coenzyme $\mathrm{A}$ as the acetyl group donor and it was shown to regulate transcription factors, molecular chaperones, effectors and cytoskeletal proteins [58]. Many of these processes are crucial for the proper functions of the neurons. For example, it was shown that lysines acetylation is relevant in the turnover of huntingtin, a protein whose mutations cause its aggregation and are the cause of the incurable neurodegenerative disorder Huntington's disease. Huntingtin acetylation alters the protein aggregation propensity [59] and regulates protein targeting for auto-phagosomal degradation [60].
This may also be of relevance for PD, being that $\alpha$ Syn is acetylated at its lysine residues [61], but the signalling pathways that are regulated by this PTM on $\alpha$ Syn are still unclear. Overall, if DOPAL modification on protein substrates competes for acetylation signalling, many crucial cellular processes may be affected.

Interestingly, it has been observed that there is a cross-talk among ubiquitination, SUMOylation and lysines acetylation pathways and a co-regulation of substrate proteins exist [52]. If DOPAL-induced changes in the level of one of these PTMs for a certain substrate alter also one of the other pathways, the already complex picture described so far may be further convoluted and would deserve careful evaluation.

\section{DOPAL-induced enzyme inhibition (Fig. 3b)}

Protein modification by DOPAL has deleterious outcomes also for enzyme activity. In fact, any enzyme with an accessible functional cysteine or lysine in the active site could be susceptible to inactivation by DOPAL, with important upshots on the metabolic pathways of interest. For instance, a proteomic study on PC6-3 cells identified tyrosine hydroxylase (TH) as target of DOPAL [62]. Administration of DOPAL at physiologically relevant concentration $(5-50 \mu \mathrm{M}$ in the cell medium) resulted in 80 95\% of $\mathrm{TH}$ activity inhibition, as assessed by $\mathrm{TH}$ purification from cells followed by HPLC quantification of LDOPA production. The authors speculated that DOPAL induces rearrangement of enzyme conformation, by modifying lysine residues that are present within or in close proximity of the active site [46]. Since TH activity is a rate-limiting step in DA synthesis from tyrosine, DOPALdependent $\mathrm{TH}$ inhibition would indirectly exacerbate the depletion of DA release in nigrostriatal circuits and parkinsonian syndrome. More recently, DOPAL appeared to cause inhibition of GAPDH activity [47]. Also, in this paradigm, both the catechol oxidation and the aldehyde moiety were required for cysteines and lysines modification. An analogous effect has been shown for DA (at least for the Cysteine residues), which was reported to modify and functionally inhibit parkin, an E3 ubiquitin ligase with genetic correlation to early-onset of PD (PARK2 locus) [48]. It is plausible to think that inactivation of parkin through catechol-cysteine adducts might involve also the catechol moiety of DOPAL, that has been reported to be even more reactive than DA $[25,36,49]$.

\section{Downstream effects of DOPAL accumulation: oxidative} stress, mitochondrial dysfunction and cell death (Fig. 3c) Several studies based on cellular model systems confirmed time- and concentration-dependence of DOPAL cytotoxicity [24]. The direct participation of DOPAL in oxidative stress has been investigated, as DOPAL can generate radical species, i.e. hydroxyl radical, in the 
presence of $\mathrm{H}_{2} \mathrm{O}_{2}$ [35]. DOPAL catechol group has a propensity to auto-oxidise to semiquinone radicals and ortho-quinones similar to DA [39]. The resulting radical oxygen species (ROS) production (Fig. 2) is expected to exacerbate the oxidative stress in neurons, leading to DNA damages, protein cross-linking and lipid peroxidation. Interestingly, cyclooxygenase-2 (COX-2), an enzyme involved in neuroinflammation and up-regulated in the SNpc of parkinsonian brains [39], was reported to catalyze DA oxidation. In the work by Anderson and colleagues, even DOPAL was shown to be a substrate of COX-2, accelerating the oxidation of DOPAL catechol as for DA. Thus, these results reiterated a connection among different aspects of PD: endotoxic catecholamines, oxidative stress and neuroinflammation, together with the potential relevance of antioxidant effectors [63]. Superoxide dismutase (SOD) may be of interest, as it efficiently clears superoxide anion by dismutation into molecular oxygen and hydrogen peroxide, removing the oxidative agent from the cellular milieu. Indeed, a recent work demonstrated that, at least in vitro, SOD1 is able to prevent lysines modification by DOPAL and associated protein cross-linking, acting as enzymatic antioxidant [64]. Other in vitro studies revealed that antioxidants agents such as $\mathrm{N}$-acetylcysteine, glutathione and ascorbic acid could effectively modulate the level of DOPAL-modified proteins in a dose-dependent manner [37, 42].

A further analogy with DA is that also DOPAL quinones could covalently modify mitochondrial protein, possibly affecting mitochondrial physiology [50]. In the work by Kristal et al., isolated mitochondria from mouse liver were exposed to DOPAL resulting in an increased opening of the mitochondrial permeability transition pore (mPTP) at concentrations close to physiological ones $(0.125-8 \mu \mathrm{M})$ [49]. Later studies reported that DA oxidation to quinones (DAQs) induced mitochondria swelling and reduced respiratory activity, suggesting the induction of the mPTP opening [17]. An analogous effect was ascribed to DAQs derived from enzymatic oxidation of DA, specifically addressing the modulation of mPTP opening to DAQs [51]. As a consequence, both DA and DOPAL-derived quinones could be responsible for the activation of the apoptotic pathway. On the other hand, DOPAL-induced decreased cell viability was assessed by measuring Lactate Dehydrogenase (LDH) release in the extra-cellular space, which is an accepted indication of necrosis $[23,33]$.

\section{Linking the Catecholaldehyde hypothesis to aSynuclein-induced pathology}

Since the identification of $\alpha$ Syn in LBs 20 years ago and its association with some familial forms of PD, the relevance of $\alpha$ Syn in the pathogenesis of PD has been widely investigated [3]. Particular interest has been given to the downstream effects of $\alpha$ Syn aggregates accumulation on neuronal homeostasis, leading to the notion that they could impair many cellular pathways and undermine organelles integrity $[65,66]$. In this frame, several research groups focused their attention on the interplay between DOPAL and $\alpha$ Syn. Starting from the observed reactivity of DOPAL aldehyde against primary amines of lysine residues, the aim has been to investigate whether DOPAL modification on $\alpha$ Syn would affect both its aggregation properties and its proteostasis. Indeed, $\alpha$ Syn might be considered a preferential target of DOPAL for at least three reasons [45]. First, lysine accounts for $10.7 \%$ of $\alpha$ Syn sequence, which is higher than the average value (around 5\%) of the lysine fraction in synaptic proteins [45]. Most of the lysines in $\alpha$ Syn sequence are within the amino acid repeats containing the consensus motif KTKEGV, which drives the transition to the alpha-helical conformation of $\alpha$ Syn $\mathrm{N}$-terminus and the association to synaptic vesicles membranes [67]. Second, $\alpha$ Syn represents the $0.5-1 \%$ of the total soluble proteins of the brain, reaching a concentration up to $40 \mu \mathrm{M}$ in pre-synaptic terminals of neurons, where it exerts its physiological function in association with synaptic vesicles membranes [68-70]. Consistently, DOPAL is mainly generated at pre-synaptic site, where MAO on the outer mitochondrial membrane quickly clears cytosolic DA in case of anomalous dyshomeostasis [71]. The third reason that points to $\alpha$ Syn as preferential target for DOPAL, is that when in the soluble monomeric state, it is an intrinsically disordered protein with good accessibility to all its lysine residues, making any potential chemical modification more likely.

A pivotal study by Burke et al. in 2008 demonstrated that in vitro DOPAL incubation with $\alpha$ Syn monomers triggers a dose-dependent protein aggregation. Similarly, SDS-resistant aggregates of $\alpha$ Syn were detected by Western Blot in lysates from SH-SY5Y cells after administration of DOPAL in the medium. The process was observed also in vivo upon direct DOPAL injection into rat $\mathrm{SNpc}$, which resulted in dopaminergic neuron loss and accumulation of $\alpha$ Syn high molecular weight species [44]. Since then, other groups provided further insights into the DOPAL-dependent $\alpha$ Syn aggregation process. Inhibition of DA uptake into synaptic vesicles by reserpine administration to dopaminergic PC12 cells, induced DA cytosolic build-up with consequent cytotoxic accumulation of DOPAL and induction of $\alpha$ Syn oligomerization [72]. Furthermore, redox active metal ions i.e. $\mathrm{Cu}, \mathrm{Fe}, \mathrm{Mn}$, whose levels are increased in parkinsonian SNpc [73], were shown to accelerate DOPAL-induced $\alpha$ Syn oligomerization in PC12 cells [74]. On the same ground, in vitro assays revealed a modulating effect of $\mathrm{N}$-terminal acetylation and familial mutations (A30P, 
A53T, E46K, G51D, H50Q) on DOPAL-induced $\alpha$ Syn oligomerization [75].

More studies were conducted by Follmer and colleagues in 2015 and by our group in 2017 [38, 41]. The former authors identified by mass spectrometry the lysine residues of $\alpha$ Syn that seem to be preferentially modified by DOPAL upon in vitro incubation. These modification sites were mainly located at the lysine-enriched $\mathrm{N}$-terminus of $\alpha$ Syn. Coherently, our experiments revealed overlapping results in vitro, but with the observation of additional modification sites involving lysine residues in the C-terminal domain upon formation of the $\alpha$ Syn-DOPAL adduct within cells. DOPAL modification of $\alpha$ Syn lysines dramatically alters $\alpha$ Syn biochemical and biophysical properties, increasing its hydrophobicity at the expense of the positive charges. Moreover, in vitro analysis revealed that DOPAL triggers $\alpha$ Syn aggregation leading to annular-shaped offpathway oligomers, which do not convert to fibrils [41].
A coherent mechanism can be proposed (as illustrated in Fig. 4), based on the observed functional effects of the reaction between $\alpha$ Syn and DOPAL on synaptic vesicles and accounting for the degeneration of the dopaminergic synapse. An increased level of DOPAL at presynaptic site promotes the covalent modification of $\alpha$ Syn. DOPAL- $\alpha$ Syn monomers exhibit reduced affinity for membrane binding [38], shifting the equilibrium toward an increased fraction of cytoplasmic $\alpha$ Syn-DOPAL, thus exacerbating $\alpha$ Syn aggregation. A further consequence is that alterations in the levels of the membrane-bound fraction of $\alpha$ Syn dramatically impair its synaptic physiological function, as $\alpha$ Syn modulates both vesicles clustering and exocytotic events [41, 77-79]. In addition, we proposed that DOPAL-modified $\alpha$ Syn oligomers might be able to form aggregated oligomers that permeabilize the membrane of synaptic vesicles, thus inducing the

MAO increased activity:

- Stimulation by truncated aSyn

- Up-regulation with age

- Neuroinflammation-induced up-regulation

- MAO polymorphisms

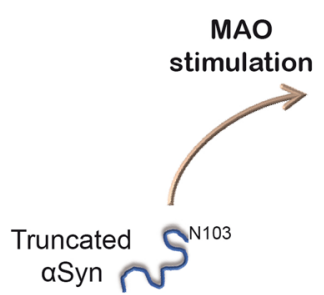

Cytosolic
dopamine

Dopamine leakage:

- DOPAL-aSyn pore-forming oligomers

- VMAT2 reduced activity

- VMAT2 loss-of-function polymorphirms

- VMAT2 inhibition by Reserpine

- Impaired synaptic vesicles endocytosis

in LRRK2-G2019S mice

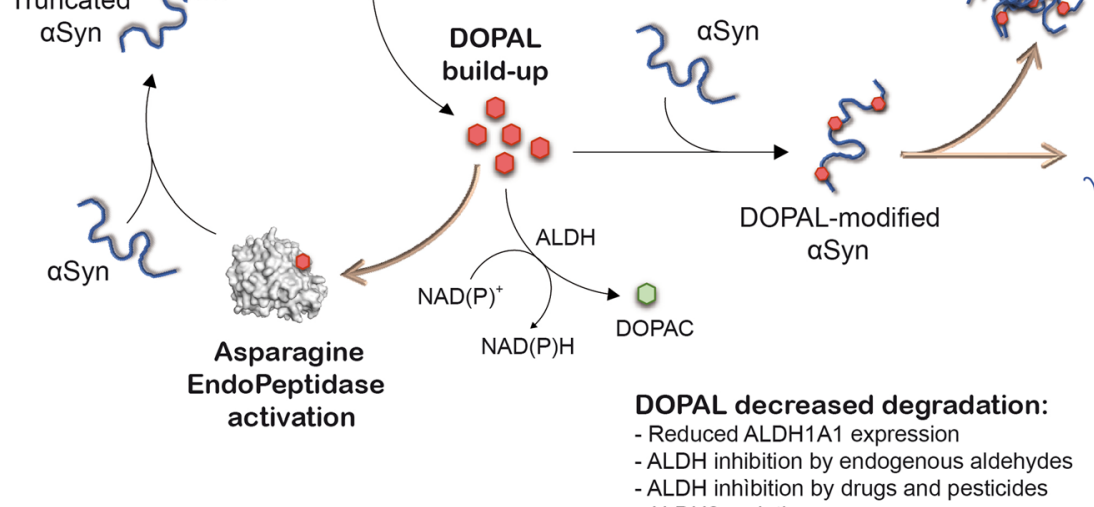

- ALDH2 variations

Fig. 4 Potential interplay between DOPAL and aSynuclein at pre-synaptic terminals and determinants of DOPAL accumulation. DOPAL accumulation at the pre-synaptic terminals covalently modifies aSyn lysines, reducing aSyn affinity for membrane binding and resulting in synaptic vesicles pools redistribution [38, 41]. aSyn-DOPAL oligomers accumulate and permeabilize synaptic vesicles membrane [41], leading to cytosolic DA release, which is further metabolized into DOPAL by MAO. Also, DOPAL activates AEP (PDB: 4aw9, in the figure), which cleaves aSyn at N103 [76]. Truncated aSyn is more prone to aggregation and stimulates MAO activity. Hence, the result is a positive loop that self amplifies, leading to aSyn aggregation and synapse degeneration. In the figure, the black thin arrows indicate the chemical reactions, while the thicker ones highlight the cellular processes. Among the factors that could lead to DOPAL build-up, the critical hubs are the dysfunction of DA storage in synaptic vesicles, increased rate of DA degradation by MAO and decreased DOPAL detoxification by ALDHs. For each point, the evidences are listed in the figure 
release of DA in the cytoplasm, that will be in turn metabolized by MAO into more DOPAL [41]. Taken together, all these events would establish a toxicity self-amplifying loop, which leads to synaptic degeneration. In addition, a very recent study highlighted a potential role for the activity of asparagine endopeptidase (AEP). AEP is reported to be highly activated in PD patients' brain where it can generate a truncated form of $\alpha$ Syn [80]. Interestingly, the resulting N103-truncated $\alpha$ Syn was shown to stimulate MAO-B activity, leading to increased rate of DOPAL production. Not only, DOPAL itself was observed to interact with and stimulate AEP, establishing an additional trail in the noxious cycle described above [76].

The functional implications of the DOPAL-induced $\alpha$ Syn dyshomeostasis at the synapses merit careful consideration. Evidence from the literature suggests a role of $\alpha$ Syn in modulating synaptic vesicles clustering, SNARE complex assembly, vesicles docking at the active zone and opening of the exocytotic fusion pore [77, 79, 81]. Importantly, both the triple knock-out mouse lacking the three synuclein isoforms (alpha, beta and gamma - Syn-TKO) and the $\alpha$ Syn-overexpressing mouse model ( $\alpha$ Syn-OVX) display alterations in the synapse architecture and neurotransmitter release. In particular, the $\alpha$ Syn overexpression results in impaired vesicles clustering with reduced vesicles density at the active zone; fast and incomplete exocytotic fusion pore dilation and pore closure; consequent decreased DA release in the striatum, weakening the nigrostriatal pathway $[77,79,82]$. Conversely, in the SynTKO mouse, more pronounced DA release was detected, potentially due to an accumulation of synaptic vesicles in the ready-releasable pool, prolonged exocytotic fusion pore dilation, faster neurotransmitter release and pore closure $[79,83,84]$. Taken together, these observations lead to the concept that although $\alpha$ Syn is not a limiting factor in the synapse activity, it is essential for maintaining the proper balance in neurotransmitter release and synaptic vesicles distribution. On this ground, since DOPAL modification of $\alpha$ Syn prevents its association to synaptic vesicle membrane, it could be considered a KO-like phenotype [38]. Yet, the observed DOPAL-induced synaptic vesicles redistribution, from ready-releasable pool to resting pool, together with $\alpha$ Syn accumulation may lean towards an overexpression-like scenario [41]. However, as DOPAL modification of $\alpha$ Syn lysines also triggers its aggregation, it may affect both synaptic vesicles mobility, docking, exocytosis and endocytosis. In addition, the pore-forming activity of the DOPAL$\alpha$ Syn oligomers increases the complexity of the scenario, placing the DOPAL- $\alpha$ Syn interplay on a different level beyond the one where the Syn-TKO and the aSyn-OVX models are set, as exemplified in Fig. 5.
Hence, future investigations will be of interest to better define the impact of DOPAL on $\alpha$ Syn homeostasis in the light of the synaptic mechanisms that $\alpha$ Syn influences.

Considering the neurotoxic potential of DOPAL and of DOPAL- $\alpha$ Syn oligomers, the spreading of those species from dopaminergic neurons could be detrimental for the surrounding environment. In this frame, our group recently demonstrated that DOPAL- $\alpha$ Syn oligomers could be secreted in the extra-cellular space by the exosomal pathway and further up taken by recipient cells [85]. Following incubation with DOPAL-modified $\alpha$ Syn containing exosomes, mouse primary cortical neurons displayed significantly higher neurite retraction, redistribution of synaptic vesicles pools and reduced levels of synaptic markers (synaptophysin and PSD-95) compared to incubation with $\alpha$ Syn containing exosomes. Interestingly, DOPAL has been reported to be transmissible from dopaminergic neurons to glial cells, where it can further enhance oligomerization of endocytosed $\alpha$ Syn [86]. This is relevant not only for PD, but also for Multiple System Atrophy (MSA), another $\alpha$ Syn-mediated pathology characterized by reduced DA in the striatum and $\alpha$ Syn cytoplasmic inclusions within oligodendrocytes $[86,87]$. These studies highlight the need of further evaluating the effective role of DOPAL in the progression of neurodegenerative processes, to identify the mechanisms of DOPAL-modified $\alpha$ Syn release and uptake, as well as their impact on both neuronal and glial physiology.

\section{Key players in the Catecholaldehyde hypothesis}

One of the crucial issues to be unravelled is the cause of the observed DOPAL build-up in parkinsonian brains, even though diverse independent mechanisms could intervene in exacerbating the toxic scenario we described. Among them, the critical hubs are the dysfunction of DA storage in synaptic vesicles, as it would result in cytosolic DA build-up, the raised DOPAL production and the increased risk of catechol oxidation; moreover, an aberrant DOPAL accumulation due to an altered metabolism, in terms of increased rate of DA deamination and decreased DOPAL oxidation (Fig. 4).

A proper DA storage in synaptic vesicles is a fundamental step in preserving pre-synaptic terminal functionality, as it assures the availability of readyreleasable neurotransmitter in the synapse and it also prevents DA auto-oxidation and radical species production. However, some PD-related conditions might compromise this event. As mentioned above, DOPAL itself generates pore-forming $\alpha$ Syn oligomers, which can in turn jeopardize synaptic vesicles integrity and induce DA leakage in the cytoplasm [41]. Moreover, the DA transporter VMAT2 is known to be involved in PD pathology, as its activity was found 


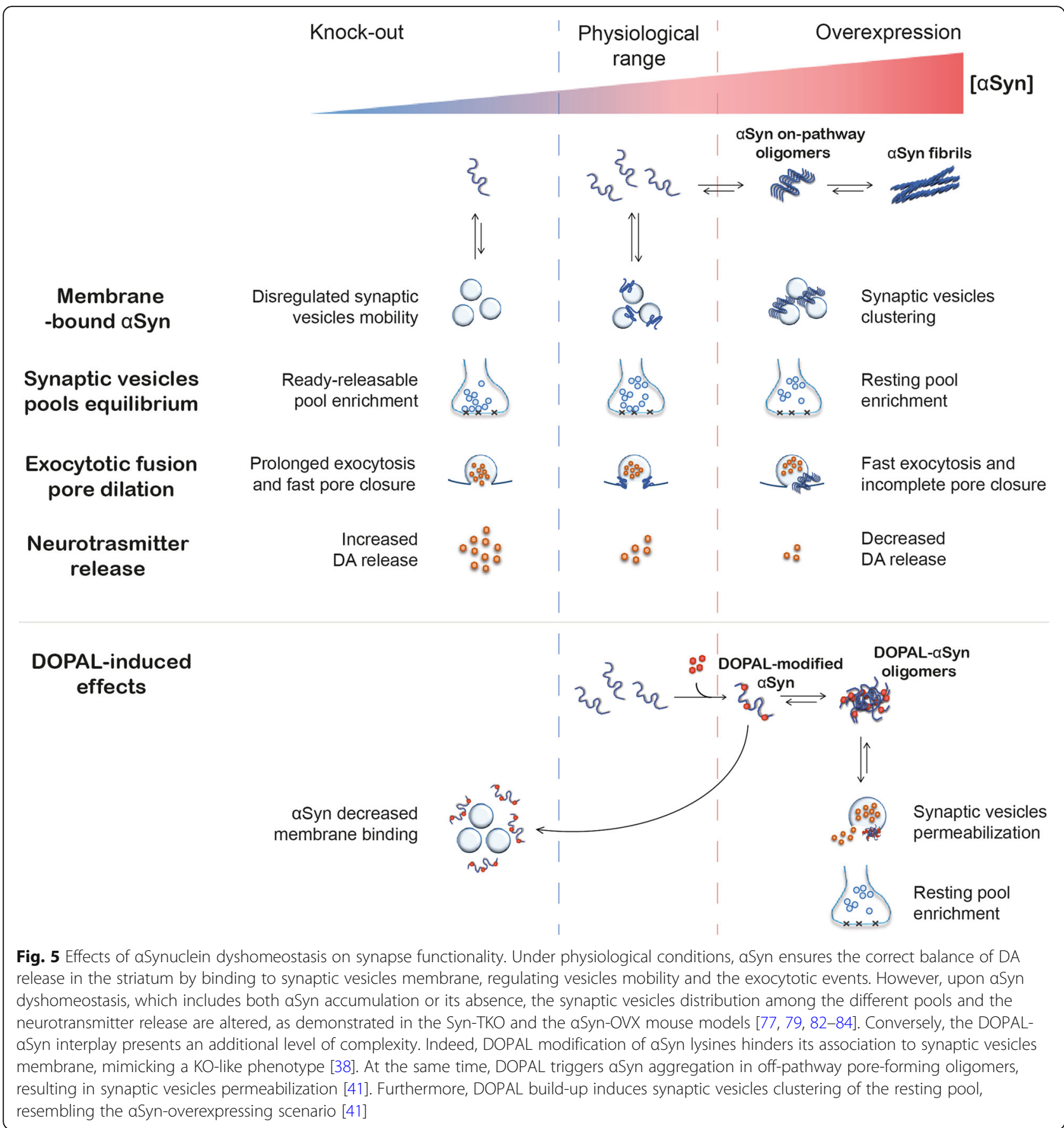

to be reduced of about $90 \%$ when DA uptake was assessed in DA storage vesicles isolated from post mortem PD patients' SNpc compared to healthy patients [88]. Consistently, a mouse model expressing only $5 \%$ of the functional VMAT2 displayed nigrostriatal degeneration and increased $\alpha$ Syn immunoreactivity in SNpc [89]. Similar results were obtained in rodent models of PD after administration of reserpine, a drug used against high blood pressure and a well-known VMAT2 inhibitor [90-92]. Also, two polymorphisms in the promoter of the Vmat2 gene (rs363371 and rs363324) were recently associated to $\mathrm{PD}$ in a case-control study in an Italian subpopulation (704 PD patients versus 678 healthy people, $p<0.01$ ) [93]. Recently, a PD-linked mutant form of Leucine-rich repeat kinase 2 (LRRK2) G2019S has been shown to affect synaptic vesicles endocytosis in patient-derived dopaminergic neurons, leading to cytoplasmic accumulation of DA and related oxidized catechols, as well as increased levels of $\alpha$ Syn [94]. 
Being such a reactive molecule, DA levels should be constantly under control. This implies that even the catabolic pathway, with MAO enzyme in the first line, plays a key role in keeping the DA at equilibrium. MAO-A and MAO-B isoforms are both expressed in $\mathrm{SNpc}$ neurons and involved in DA metabolism, although MAO-B is reported to be mainly expressed in astrocytes [95]. Interestingly, MAO-B has been more in the spotlight in PD research. According to different studies, MAO-B expression exponentially increases with age and it can be upregulated, for instance, in neuroinflammation [96-98]. In PD, MAO-B activity was shown to be enhanced [99]. In addition, from the genetic point of view, some variants of $M a o-B$ gene encode for an hyperactive form of the enzyme and are associated to PD cases [100-102]. Correspondingly, a mouse model with an inducible overexpression of MAO-B in astrocytes recapitulates many features of parkinsonian phenotype i.e. dopaminergic neuronal loss, oxidative stress, motor phenotype, $\alpha$ Syn altered proteostasis, astrogliosis and microglia activation [103]. These observations, together with the preferential expression of MAO-B in astrocytes, highlighted the importance of maintaining DA homeostasis, both in neurons, glial cells and the extra-cellular environment. Finally, it is worth reminding of the interplay among DOPAL, AEP, $\alpha$ Syn and MAO-B, which underlines an indirect positive feedback of MAO stimulation by its substrate DOPAL [76, 80].

Other relevant players are ALDHs, which are the main enzymes involved in DOPAL degradation. Any kind of inefficiency of these enzymes would result in a detrimental DOPAL build-up in nigrostriatal neurons, however ALDHs have been less investigated in the PD background. In the following paragraphs of this review, we will particularly focus on ALDH enzymes, with the aim to explore their potential role and impact in SNpc dopaminergic neurons susceptibility in PD.

\section{DOPAL detoxification by aldehyde dehydrogenases in Parkinson's disease}

DOPAL is physiologically degraded by two different pathways: oxidation by ALDH and reduction by ALR/ AR (Fig. 1). Although these different enzymes are all expressed in the neurons of the substantia nigra, DOPAL degradation primarily occurs through a $\mathrm{NAD}(\mathrm{P})^{+}$-dependent irreversible oxidation by ALDH to DOPAC, a much less reactive catabolite in which the aldehyde moiety is converted to a carboxyl group [24]. The human ALDH superfamily includes 19 functional genes, encoded in distinct chromosomal locations. Most ALDHs have wide tissue distribution and diverse substrate specificity [104], however only ALDH1A1 and ALDH2 are responsible for DOPAL degradation in dopaminergic neurons of SNpc [24]. ALDH1A1 is expressed in the brain, eye lens, retina, lung, liver, kidney and testis, while ALDH2 is constitutively expressed in the mitochondrial matrix of various tissues, namely liver, kidney, lung, heart and brain [104]. Of note, ALDH1A1 is the most represented cytosolic form in SNpc dopaminergic neurons, being expressed both in axons and neuronal terminals [40, 105-107]. In nigral neurons, ALDH1A1 expression starts at the early stages of development under the transcriptional control of the Pairedlike homeodomain 3 (Pitx3) transcription factor, shortly after the initial expression of $\mathrm{TH}$ which defines the dopaminergic phenotype of this particular type of neurons [105, 108]. A study by Liu et al. in 2014 described a unique distribution of ALDH1A1 expression in SNpc, which seems to be conserved both in mouse and human brain. According to the reported histological analysis, ALDH1A1 is present only in the ventro-lateral tier of SNpc whose axons project predominantly to the rostral dorsal striatum, unveiling the existence of two distinct class of nigral dopaminergic neurons [40, 109].

Both ALDH1A1 and ALDH2 exist as tetramer with 501 amino acids each subunit and they share $68 \%$ of sequence identity (ALDH2 has an additional transition peptide at the $\mathrm{N}$-terminal which targets the protein to mitochondria). As shown by the superimposed 3D structures in Fig. 6, the two proteins display high level of structural similarity, both for the single subunit and for the spatial orientation of the conserved amino acids of

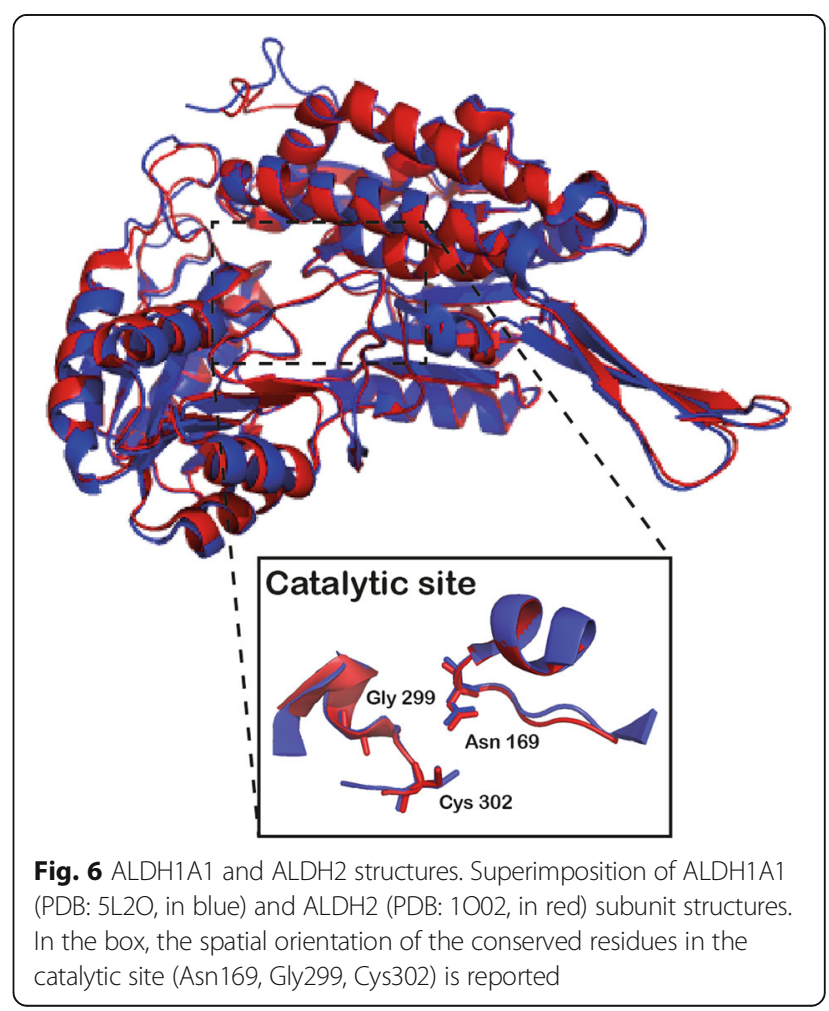


the catalytic site. ALDH1A1 best known substrate is retinaldehyde $\left(\mathrm{K}_{\mathrm{m}}<0.1 \mu \mathrm{M}\right)$, whose oxidation leads to retinoic acid (RA) production. RA is required for differentiation and development of dopaminergic neurons [108]. Moreover, ALDH1A1 was demonstrated to metabolize -aminobutyraldehyde and further mediate an alternative synthesis pathway of GABA, which can be co-released with DA and displays an additional inhibitory modulation at post-synaptic level in the striatum $[107,110-112]$. Of note, the GABA release was demonstrated to be limited to only a subset of nigral neurons, which is consistent with the differential expression of ALDH1A1 in subpopulations of SNpc dopaminergic neurons $[40,110]$ and may suggest a role for this other neurotransmitter in the PD-vulnerability observed in certain dopaminergic neurons. Instead, ALDH2 is primarily involved in acetaldehyde oxidation during ethanol metabolism. Both ALDH1A1 and ALDH2 were also invoked for detoxification of aldehydes derived from lipid peroxidation, i.e. 4-hydroxynonenal (4-HNE) and malondialdehyde (MDA) [104]. Limited data are available on DOPAL as a substrate for ALDHs. Most of the recent literature refers to a review by Marchitti et al. 2007, where the values of the affinity constants of DOPAL for ALDH are reported [24, 113, 114]. These data were obtained by in vitro enzymatic assays, performed using ALDH proteins purified from human, rat or rainbow trout both from liver and brain. The highest affinity values are reported for ALDH1A1 and ALDH2 purified from human liver, with a calculated $K_{m}$ of $0.4 \mu \mathrm{M}$ and $1.0 \mu \mathrm{M}$, respectively. Although ALDH9A1 affinity for DOPAL is comparable to ALDH1A1 and ALDH2 $\left(K_{m}\right.$ of $\left.2.6 \mu \mathrm{M}\right)$, it should be mentioned that the nigral expression of this enzyme is quite low $[24,106]$. More recently, Cai et al. reported that in mouse SNpc dopaminergic neurons both ALDH7A1 and ALDH1A1 are expressed and the two enzymes share $91 \%$ of protein identity [106]. Hence, ALDH7A1 is likely to be recruited (in addition to ALDH1A1) for DOPAL degradation in mouse SNpc dopaminergic neurons, contributing to a proper DA catabolism. Even though an Aldh7a1 homo$\log$ is found in human genome, the human Aldh1a1 and Aldh7a1 genes are located in different chromosomes and no expression of ALDH7A1 has been reported in adult human brain [104]. This would imply a higher vulnerability of the human SNpc dopaminergic neurons to DOPAL toxicity due to the lack of ALDH7A1 expression and therefore reduced overall efficiency in DOPAL degradation. On the contrary, one may speculate that the presence of additional ALDH cytosolic isoenzymes in the mouse SNpc neurons may counteract DOPAL buildup when ALDH1A1 is lacking.

Interestingly, both ALDH1A1 and ALDH2 are also expressed in the dopaminergic neurons of the ventral- tegmental area (VTA) and responsible for DOPAL detoxification [7, 40, 107]. Also, in both SNpc and VTA the differential topographic distribution of ALDH1A1 expression in the ventro-lateral tier compared to the dorso-medial one is conserved, together with the pronounced age-dependent degeneration of the ALDH1A1negative neurons observed in the transgenic A53T mouse model [40]. On this ground, it becomes intriguing to unravel the molecular mechanisms behind the differential degeneration of SNpc and the VTA in PD. In their review, Brichta and Greengard made an accurate comparison between the dopaminergic neurons in the two areas, highlighting a series of molecular determinants potentially involved i.e. electrophysiological elements, energy demand, transporters, receptors, enzymes [7]. Among them, ALDH1A1 was discussed, even though its role was not clearly depicted as the main reported arguments were based on the observation of the work by Liu [40]. However, a previous paper investigated the ALDH1A1 mRNA levels in SNpc and VTA in post mortem PD patients' brain, revealing a significant decrease in the transcript level in the SNpc but not in the VTA [115]. At this point, further investigations on the expression of other ALDHs or ARs/ALRs in the VTA would help in determining the efficiency of DOPAL degradation in the VTA. This would explain the observed reduced degree of vulnerability of the VTA in PD compared to the SNpc, where ALDH1A1 appears to be the major protective factor against DOPAL neurotoxicity. Based on the available reports, we suggest that the relative levels and activity of the different ALDHs or ARS/ ALRs may participate in setting the threshold that makes specific brain regions more vulnerable to PD; therefore, a comprehensive study on these enzymes in PD models and patients is desirable to unravel this issue.

In Table 1, the most relevant features of the ALDH1A1 and ALDH2 are summarized.

\section{Aldehyde dehydrogenases as downstream targets in Parkinson's disease}

In the last decades, several studies reported alterations in ALDHs expression and activity levels in PD patients' nigral tissues, providing further support to the DOPAL paradigm for neurodegeneration. Initial evidence came from oligonucleotide in situ hybridization experiments on human post-mortem midbrain from PD patients with unreported aetiology. Aldh1a1 mRNA was found markedly reduced in TH-positive neurons in SNpc of parkinsonian brains compared to controls [115]. A following genome-wide transcriptomic assay on PD patients confirmed similar down-regulation of Aldh1a1 mRNA in SNpc together with other 139 genes, revealing alterations in ubiquitin-proteasome, heat shock proteins, iron and oxidative stress regulated proteins, cell adhesion/ 
Table 1 Comparison between ALDH1A1 and ALDH2, in terms of expression, biochemistry and PD-related aspects

\begin{tabular}{|c|c|c|}
\hline & ALDH1A1 & $\mathrm{ALDH} 2$ \\
\hline Tissue expression & $\begin{array}{l}\text { Brain, eye lens, retina, lung, liver, kidney, } \\
\text { testis [104] }\end{array}$ & Liver, kidney, heart, lung, brain [104] \\
\hline Subcellular localization & Cytosol $[40,105,106]$ & Mitochondrial matrix [104] \\
\hline Substrates & $\begin{array}{l}\text { Retinaldehyde }\left(k_{m}<0.1 \mu \mathrm{M}\right)[116] \\
\text { DOPAL }\left(k_{m} 0.4 \mu \mathrm{M}\right)[24,113,114] \\
\text { 4-HNE }\left(k_{m} 4.8 \mu \mathrm{M}[117] ; 17.9 \mu \mathrm{M}[118]\right) \\
\text { MDA (km } 3.5 \mu \mathrm{M}[117] ; 114.4 \mu \mathrm{M}[119]) \\
\text { Y-aminobutyraldehyde }(800 \mu \mathrm{M})[112]\end{array}$ & $\begin{array}{l}\text { Acetaldehyde }\left(k_{m}<1 \mu \mathrm{M}\right)[120] \\
\text { DOPAL }\left(k_{m} 1 \mu \mathrm{M}\right)[121] \\
\text { 4-HNE and MDA [122-124] } \\
\text { Y-aminobutyraldehyde }(500 \mu \mathrm{M}) \text { [112] }\end{array}$ \\
\hline \multicolumn{3}{|l|}{ PD-related } \\
\hline Genetic variants & N.A & $\begin{array}{l}\text { - Haplotype: rs737280; rs968529; rs16941667; } \\
\text { rs16941669; rs9971942 (California) [125] } \\
\text { - Haplotype: rs4767944; rs441; rs671 (China) [126] } \\
\text { - rs671 SNP (China) [127] }\end{array}$ \\
\hline \multirow[t]{2}{*}{ Expression levels } & $\begin{array}{l}\text { Reduced mRNA levels: } \\
\text { - TH-positive neurons in PD patients' } \\
\text { brain [128] } \\
\text { - transgenic A53T mouse striatum [129] }\end{array}$ & N.A. \\
\hline & $\begin{array}{l}\text { Decreased protein levels: } \\
\text { - PD patients' brain [130, 131] } \\
\text {-LRRK2-G2019S knock-in mouse DA neurons [132] }\end{array}$ & \\
\hline \multirow[t]{3}{*}{ Enzyme inhibition * } & $\begin{array}{l}\text { Epidemiological studies: } \\
\text { - traces of Dieldrin in tissues of exposed PD } \\
\text { patients [133] } \\
\text { - Benomyl exposure correlates with PD risk [134] }\end{array}$ & \\
\hline & $\begin{array}{l}\text { In vitro: } \\
\text { - 4-HNE and MDA }[135,136] \\
\text { - DOPAL }(>5 \mu \mathrm{M})[121,136] \\
\text { - Benomyl }[134]\end{array}$ & \\
\hline & $\begin{array}{l}\text { Cellular models of ALDH inhibition: } \\
\text { - rat purified synaptosomes treated with 4-HNE } \\
\text { and MDA [34] } \\
\text { - SH-SY5Y cells treated with Disulfiram [137] } \\
\text { - Neurons from Daidzin administered hamster [138] } \\
\text { - PC6-3 cells treated with Dieldrin [139] } \\
\text { - primary neurons and SK-N-MC cells treated } \\
\text { with Benomyl [134] }\end{array}$ & \\
\hline \multirow[t]{2}{*}{ In vivo models * } & 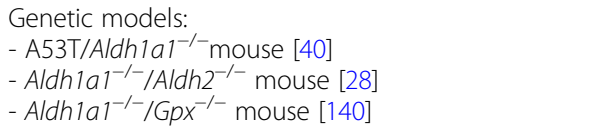 & \\
\hline & $\begin{array}{l}\text { Toxin-based models: } \\
\text { - Benomyl intraperitoneally administered mouse [141] } \\
\text { - Benomyl exposed zebrafish embryos [134] } \\
\text { - Ziram exposed zebrafish embryos [142] }\end{array}$ & \\
\hline
\end{tabular}

"The "Enzyme Inhibition" and "In vivo models" sections refer to both ALDH1A1 and ALDH2

cellular matrix and vesicles trafficking genes [143]. Of note, neither study reported alterations in Aldh2 mRNA levels.

Coherently with the transcriptomic analysis, also histological data on midbrain from sporadic PD patients' samples revealed a reduced ALDH1A1 immunoreactivity in dopaminergic neurons in PD patients [130]. These results were followed by a tissue-based comparative proteome study of PD SNpc from human post-mortem brains. Decreased expression of ALDH1A1 was found both in familial and idiopathic PD samples, compared to controls [131]. In addition, indirect evidence of decreased ALDH activity in PD emerged from a quantification of catechols in PD patients' brain. More precisely, the DOPAC:DA ratio, assumed to depend on ALDH activity, was found to be significantly reduced in PD's putamen [27]. In parallel, giving the lower expression of ALDH1A1 in PD, another study was designed to evaluate mitochondrial ALDH2 activity in sporadic PD. ALDH2 was purified from the frontal cortex and putamen of PD patients' brain and its activity was quantified by an in vitro colorimetric assay. ALDH2 activity resulted significantly elevated in PD putamen compared to controls, while in the frontal cortex 
there was no detectable difference [144]. Given that ALDH2 is involved in the metabolism of endogenous and exogenous toxic aldehydes, the increased activity in PD putamen might reflect the higher demand in DOPAL and lipid peroxidation-derived (i.e. 4-HNE) detoxification.

Taken together, these observations point at ALDH as one of the potential players of PD-related pathology. This role is also substantiated by the observed effects of ALDH inhibition by two different classes of molecules [139]. The first one includes endogenous catabolic aldehydes, with emphasis on those derived from oxidative stress and lipid peroxidation, events recurrently associated to PD pathogenesis. Indeed, protein adducts of 4$\mathrm{HNE}$ are enriched in SNpc neurons of PD patients [145]. As mentioned before, both 4-HNE and MDA are substrates of ALDH1A1 and ALDH2 themselves. However, it was also demonstrated that high concentrations of lipid peroxidation products induce ALDH activity inhibition [34, 135, 136]. Treatment of synaptosomal proteins with 4-HNE and MDA resulted in dose-dependent ALDH inhibition and consequentially decreased DOPAL degradation. This, in turn, led to accumulation of DOPAL and DOPAL-modified proteins, as detected by NBT staining [34]. Interestingly, DOPAL itself has been demonstrated to act as inhibitor of ALDH in vitro at concentrations higher than $5 \mu \mathrm{M}$, due to covalent modification of amino acids important for enzyme activity [121, 136].

The second class of ALDH inhibitors includes several drugs, environmental agents and chemical compounds [146]. Among used drugs, the anti-alcohol abuse disulfiram is a potent irreversible inhibitor of both ALDH1A1 and ALDH2 as its metabolic products specifically modify Cys302, a conserved residue in the catalytic site [146]. Indeed, disulfiram treatment on catecholaminergic SH-SY5Y cells, together with DOPAL synthesis stimulation by DA administration, led to cellular death over time [137]. On the same line, inhibition of ALDH1A1 by disulfiram resulted in decreased GABA synthesis and release, leading to an altered post-synaptic inhibitory modulation [107]. Similarly, daidzin, another drug used against alcohol dependency, was showed to inhibit ALDHs in hamsters with consequent accumulation of biogenic aldehydes as DOPAL and 5-hydroxyindole-3-acetaldehyde [138]. Later, chemicals used in agriculture were also found to inhibit ALDHs. These includes the organochlorine pesticide dieldrin, which induced a dose-dependent DOPAL accumulation in PC6-3 cells as well as oxidative stress, alterations in DA trafficking and metabolism, mitochondrial dysfunction and apoptosis [139]. Dieldrin was used as pesticide and insecticide during the second half of the XX century and was then banned in the late 90s due to its potential carcinogenic activity. Of relevance here, elevated dieldrin levels were also detected in exposed
PD patients, compared to controls $(p=0.005)$ [133, 147]. Another important epidemiological study by Fitzmaurice et al. in 2013 provided robust in vivo evidence of ALDH inhibition by Benomyl as causative potential factor of PD. Benomyl, indeed, is a benzimidazole fungicide widely used in agriculture until a correlation with liver tumours, brain malformations and reproductive defects was established. At molecular level, once it is metabolized in cells, one of its by-products becomes a strong irreversible inhibitor of ALDH2 due to carbamoylation of Cys302 in the active site [146]. Also, it has been shown to impair microtubule dynamics and to inhibit the ubiquitin-proteasome system. In the work by Fitzmaurice, a positive correlation between benomyl exposure and PD occurrence was reported. Analysis of 360 PD patients and 754 normal subjects, allowed to calculate a PD risk of around $67 \%$ for individuals with ambient benomyl exposure ( $p=0.0027)$ [134]. In addition, in vivo studies on zebrafish embryos exposed to the fungicide displayed fewer VMAT2-positive neuronal clusters and an altered swimming behaviour. In vitro experiments confirmed benomyl selective cytotoxicity in mouse primary dopaminergic neurons and $\mathrm{ALDH} \mathrm{IC}_{50}$ was measured to be $0.12-0.14 \mu \mathrm{M}$ when assayed on isolated mitochondria from rat liver [134]. Zebrafish embryos were also used as in vivo model to prove the pathogenic implications of ziram, another pesticide, in PD [142]. Epidemiological studies determined a $80 \%$ of increased risk to develop PD in workplace exposure to ziram and paraquat [148]. From the molecular point of view, ziram causes inhibition of the proteasome, aggregation of $\alpha$ Syn and cell death, with particular effect on dopaminergic neurons [149]. Although it was not clearly specified, being a dithiocarbamate like other ALDHs inhibitors (i.e. disulfiram), ziram might share similar ability to alter DA metabolism toward DOPAL increase and trigger the selective $\alpha$ Syn-induced toxicity in SNpc dopaminergic neurons in PD. It is worth to mention that only some of the studies that aimed to investigate the inhibition mechanisms we described specifically refer to the ALDH2 form as the target. However, in most cases the inhibition is not specific, and it is likely to affect also ALDH1A1 (Table 1).

Finally, beside ALDHs inhibitors, other molecules might have indirect negative effects on ALDHs activity. For instance, the potent neurotoxin methylmercury (MeHg) is known to impair dopamine homeostasis and to cross the blood-brain-barrier [150]. MeHg treatment on dopaminergic PC12 cells resulted in increased DA synthesis and release, but also DOPAL accumulation. Although $\mathrm{MeHg}$ did not inhibit ALDH enzyme, it induced depletion of $\mathrm{NAD}^{+}$cellular reservoir, which is the required cofactor for ALDH activity [151]. More generally, any stimulus which affects $\mathrm{NAD}^{+}$reservoir at mitochondrial level would result in decreased ALDHs activity and impaired DOPAL detoxification. Given the fact that 
dysfunction of complex I is an important event in PD pathogenesis, it has been reported that inhibition of complex I and III of the mitochondrial respiratory chain resulted in increased levels of DOPAL and DOPET [152].

The deregulation of ALDH expression might occur also at transcriptional level. Cai's group has been particularly active in studying ALDH functional role and expression in dopaminergic neurons of SNpc. In the last few years, they aimed to investigate ALDH1A1 relation to PD pathogenesis by using mouse models with mutations in diverse PD loci. First, they developed a new line of tetracycline-regulated inducible transgenic mice with the over-expression of the human form of $\alpha$ Syn carrying the pathological mutation A53T in dopaminergic neurons [129]. Those mice revealed a marked motor phenotype, decreased DA release and impairment in various cellular pathways. Focusing on ALDH1A1, both transgenic and non-transgenic mice show age-dependent decrease in ALDH1A1 expression and both ALDH1A1 protein and Aldh1a1 mRNA levels were significantly lower in A53T transgenic mice striatum. These data prompted the hypothesis that both age and pathogenic $\alpha$ Syn overexpression may suppress Aldh1a1 expression in dopaminergic neurons [40]. This working hypothesis hinges on the observation that overexpression of the human disease-causing form of $\alpha$ Syn appeared to promote proteasome-dependent degradation of nuclear receptorrelated 1 (Nurr1) protein, a developmental transcriptional factor which is involved in midbrain dopaminergic neurons differentiation [129, 153]. Interestingly, Nurr1 has been demonstrated to directly regulate Pitx3, an upstream promoter of Aldh1a1 gene transcription [108, 154]. Moreover, histological studies on human SNpc revealed age-dependent down-regulation of Nurr1 [155]. All things considered, it is plausible to think that age and pathological $\alpha$ Syn accumulation may progressively decrease ALDH1A1 expression by affecting Nurr1 and consequently Pitx3. In addition, histological studies on A53T transgenic mice's brain highlighted that the dorso-medial tier of $\mathrm{SNpc}$, whose neurons do not express ALDH1A1, showed increased susceptibility to $\alpha$ Syn-induced pathology, suggesting a protective role by ALDH1A1 [40].

Cai's group also developed a transgenic mouse model expressing either the wild-type human LRRK2 or the gain-of-function mutant form LRRK2-G2019S in midbrain dopaminergic neurons [132]. Although no motor phenotype or midbrain degeneration were observed in LRRK2-G2019S mice, the dopaminergic pathway was affected. Indeed, age-dependent decreased expression of TH, VMAT2, DA transporter (DAT) and ALDH1A1 were revealed, together with reduced Pitx3 transcript and protein levels. In line with the considerations mentioned above, the authors investigated Nurr1 protein levels, which resulted in the same age-dependent downregulation in LRRK2-G2019S mice, while wild-type mice did not show the analogous pattern. Thus, they speculated that LRRK2 might be involved in the regulation of Nurr1 and Pitx3 proteostasis, even if they did not provide any direct evidence. Instead, opposite results were recently obtained by studying the nigro-striatal dopaminergic pathway in LRRK2-G2019S knock-in mice, where no alteration was detected compared to wild-type mice [19]. To our knowledge, these are the only independent studies aimed to investigate the role LRRK2 in regulating the dopaminergic pathway. However, the available evidence to date is not sufficient to draw a conclusion.

Starting from the observations of a potential implication of ALDHs decreased expression and activity in PD, an attempt to rescue the ALDH loss-of-function pathogenic condition has been made. A recent work on a rotenone-induced PD model in SH-SY5Y cells proposed wild-type ALDH2 overexpression or enzyme activation as neuroprotective factors against rotenone-induced mitochondria dysfunction and cell death [156]. This was achieved by treating cells with Alda- 1 (N-(1,3-benzodioxol-5-ylmethyl)-2,6-dichloro-benzamide), a small molecule which was previously identified as specific ALDH2 activator, acting as a molecular chaperone [157, 158]. Similarly, Alda-1 intraperitoneally administered to mice that were previously exposed to rotenone or MPTP, resulted in reduced $\mathrm{TH}$-positive neuron degeneration in mice SNpc [156].

\section{Aldehyde dehydrogenases as contributors to Parkinson's disease}

All the evidence from the literature outlined above strongly supports a contribution of ALDH alterations in DOPAL build-up and neurotoxicity. Most studies converge to a scenario in which ALDHs are a down-stream target of other pathogenic mechanism rather than a primary effector. To our knowledge, no genome-wide association study highlighted ALDH as risk gene for PD. However, very recent genetic studies opened a new line of research that again poses ALDH as potential accomplice of PD pathology. The first attempt was performed by Fitzmaurice, who tried to correlate pesticides exposure and genetic variations of Aldh to increased risk for PD [125]. In his case-control study in California, an increased risk of PD around 2- to 6-fold was correlated with the exposure to ALDH-inhibiting chemicals among a panel of pesticides. In addition, an Aldh2 haplotype (rs737280; rs968529; rs16941667; rs16941669; rs9971942) was associated to an increased PD risk in subjects who were exposed to high doses of ALDH-inhibiting compounds: metal-coordinating dithiocarbamates (i.e. maneb, ziram), imidazoles (i.e. benomyl, triflumizole), 
dicarboxymides (i.e. captan, folpet) and organochlorines (i.e. dieldrin). Of note, the mentioned haplotype did not include the single known mutation E487K of ALDH2 (ALDH2 2, the rs671 SNP), which kills enzyme activity by reducing coenzyme binding affinity [128]. The authors claimed that the variation could not be assessed as less than $2 \%$ of the considered population carried the mutant allele, resulting no statistically relevant. Indeed, ALDH2*2 mutation is mostly diffuse in East Asian population where alcohol intolerance is frequent because of the mutation. A study in Taiwan demonstrated that PD patients with rs671 SNP were more prone to develop neuropsychological and cognitive dysfunctions than patients carrying the full active enzyme [159]. Also, a genetic screening on 155 PD patients of a Chinese population confirmed a positive correlation between ALDH2*2 mutation and elevated PD risk [127]. Another epidemiological study on a Han Chinese population investigated whether some Aldh2 variations increase susceptibility to PD [126]. Considering 584 sporadic PD patients and 582 age and gender-matched controls, three main Aldh2 variants emerged (rs4767944; rs441; rs671), providing another haplotype associated to increase of PD risk. Taken singularly, only the rs4767944 variant but not the rs441 and the loss-of-function rs671 resulted as risk factor for PD. However, the same candidate Aldh2 polymorphism rs4767944 was not associated to increased risk of PD incidence when assessed in a casecontrol study in the Iranian population [160].

Taken together, these data indicate that specific variations and haplotypes of ALDHs gene may be considered as risk factors for PD. In the future, a more comprehensive investigation of both Aldh1a1 and Aldh2 gene expression would help clarify their role in PD.

\section{May aldehyde dehydrogenase-null mice be a model Parkinson's disease?}

Besides biochemical and cellular studies, some Aldhnull mice have been generated to validate the Catecholaldehyde Hypothesis (Table 1). One of them was developed by crossbreeding between their Pitx3-tTA/ tetO-A53T transgenic mice with Aldh1a1 knock-out mice [40]. The resulting A53T/Aldh1a1 ${ }^{-/-}$mice exacerbated the motor phenotype of the $A 53 T / A_{l d h}{ }^{+/+}$ mice, assessed by open-field test, rotarod test and rearing ability in 6 months-old animals. Also, A53T/ Aldh $1 a 1^{-/-}$mice exerted significant TH-positive neuron loss in SNpc compared to A53T/Aldh ${ }^{+/+}$mice. Of note, protein pull-down assay with APBA demonstrated that the absence of Aldh1a1 promoted catechol-triggered A53T- $\alpha$ Syn aggregation in primary neuronal cultures, providing an indirect indication of DOPAL build-up in SNpc dopaminergic neurons. No quantification of DOPAL and other catechols was shown, even if a previous work reported decreased
DOPAC levels and DOPAC/DA ratio in another Aldh1a1 $1^{-/-}$mouse model [161]. The Aldh1a1 knockout mouse per se did not show any parkinsonian phenotype, suggesting that the absence of just ALDH1A1 is not enough to model the Catecholaldehyde hypothesis in mice, at least in the analysed time frame. The single knock-out for the Aldh2 gene did not present an altered dopaminergic pathway, when monitored as DA and DOPAC levels [91]. The outcome is different for the double knock-out mouse for both the cytosolic and mitochondrial Aldhs, which recapitulated in its phenotype most of the parkinsonian

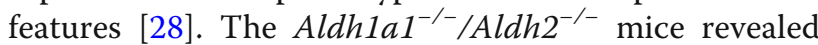
an age-dependent motor impairment, assessed by gait analysis and accelerating rotarod test on 6, 12, and 18 months old mice, as well as TH-positive neuron degeneration in SNpc. Both DA and DOPAC/DA ratio presented a significant age-dependent decrease, coherent with increasing DOPAL in the striatum. These results were confirmed in a follow up paper by

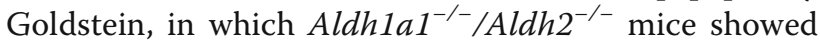
increased DOPAL, DOPET, DOPAL/DA and DOPET/ DOPAC and decreased DOPAC and DOPAC/DOPAL compared to wild-type mice [27]. The authors also compared catechols content of Aldh double knockout mice striatum with a benomyl-exposed mouse model [141]. Analysis of striatal tissue resulted in increased DOPAL (3.1 fold) and DOPET (2.5 fold) but decreased DOPAC, recapitulating catechol levels detected in the Aldh genetic model. Based on these data, Aldh $1 a 1^{-/-} /$Aldh $^{-/-}$mouse may be suggested as an interesting in vivo model for PD, but the fact that the presence of neither high molecular weight $\alpha$ Syn aggregates nor DOPAL-modified $\alpha$ Syn was reported lacks to provide a decisive evidence for the Catecholaldehyde Hypothesis. Finally, another double knock-out mouse, deficient for ALDH1A1 and glutathione peroxidase 1 (GPX1) was recently characterized [140]. The rationale behind the development of this transgenic mouse was to induce simultaneous accumulation of free radicals and reactive aldehydes, which are both primary sources of oxidative stress in neurons in PD. Indeed, both Aldh1a1 and GPX1 mRNA levels are reduced in SNpc of PD patients [162]. As expected, these mice presented a reduced DOPAC and DA/DOPAC content in striatal dopaminergic neurons, together with increased level of 4HNE-proteins adducts. Moreover, they also showed significant motor impairment as assessed by accelerating rotarod test and pole test, with trend toward agedependent worsening. Giving the complexity and variability in mouse phenotyping, a quantitative comparison of motor performance among all aforementioned transgenic mice is not trivial. Anyway, 
these mouse models provide useful tools in PD research, both to unravel molecular mechanisms driving the preferential vulnerability of $\mathrm{SNpc}$ dopaminergic neurons and to explore new therapeutic strategies.

\section{Translational implication of the Catecholaldehyde hypothesis}

Among the therapeutic strategies for PD, MAO inhibitors have been used since the 1960s and they are currently FDA approved drugs. If considered in the light of the Catecholaldehyde Hypothesis, the MAO inhibition approach sounds even more promising as it would block at least one source of DOPAL build-up. As a proof of concept, in a work by Goldstein et al. of 2016, different $\mathrm{MAO}-\mathrm{A}$ and MAO-B inhibitors were administrated to PC-12 cells, to evaluate their ability in decreasing DOPAL cellular content [163]. Coherently, clorgyline, rasagiline and selegiline resulted to be efficient in inhibiting MAO and reducing endogenous DOPAL production. Also, in another study, the MAO-B inhibitor rasagiline, being an amine itself, was demonstrated to react with DOPAL, to reduce in vitro DOPAL-induced $\alpha$ Syn oligomerization and to exert a neuroprotective effect on PC-12 cells [164]. However, potential drawbacks of MAO inhibition need to be taken into account, as increased levels of cytosolic DA might lead to decreased $\mathrm{TH}$ activity due to feedback inhibition. Conversely, if not properly stored in synaptic vesicles, DA undergoes autooxidation, resulting in oxidative stress [163]. In this case, the beneficial effect of reducing DOPAL concentration would be overshadowed by cytosolic DA neurotoxicity. It follows that the potential of MAO inhibition as disease modifiers hinge on two aspects: the first is an accurate patient stratification as more prone to develop DOPAL build-up based on ALDHs dysfunction. The second is early action, being that the DOPAL build-up and the synaptic damage likely precede neuronal death and disease manifestation.

On this ground, another strategy might be the scavenging of reactive aldehydes by an excess of amino-molecules, which would compete with protein lysines. As an example, metformin is a biguanidine molecule and an FDA-approved drug for the treatment of Type 2 Diabetes Mellitus (T2DM). Interestingly, T2DM has been recognized as a risk factor for PD [165]. Treatments with metformin were showed to have not only antidiabetic but also neuroprotective action [166]. From a molecular point of view, metformin acts on different pathways i.e. controlling mitochondrial physiology, activating the autophagic pathway and modulating neuroinflammation. It has been also demonstrated to reduce the elevation of phosphorylated $\alpha$ Syn (an accepted indicator of aSyn-related pathology [167]) by activating mTORdependent phosphatase 2A [168, 169].
Nevertheless, a more comprehensive understanding of the DA catabolic pathway and its functionality in PD patients would allow to design more targeted and effective therapeutic strategies.

\section{Conclusions}

A full description of the molecular mechanisms that lead to DOPAL build-up in parkinsonian brains is still unavailable. More likely, the combination of age, genetic predisposition and environmental factors are responsible for a possible synergistic dysregulation of several pathways, including DA metabolism, redox state homeostasis and neuronal proteostasis [22]. Further investigations on both up-stream effectors as well as down-stream outcomes of DOPAL build-up are necessary. In addition, several of the neurotoxic outcomes that have been attributed to DA so far, should be reconsidered to include the even more reactive DOPAL. More than DA, DOPAL represents a dangerous player due to the synergy between the catechol and the aldehyde moieties, increasing exponentially the detrimental consequences of impaired DA regulation. This would entirely fit with the multiplehit scenario described by Burbulla et al. [18], in which mitochondria dysfunction lead to increased oxidized catechol species, $\alpha$ Syn aberrant accumulation and failure of protein degradation systems, both in familial and idiopathic PD cases.

According to the literature reviewed in this paper, ALDH potentially represents a crucial hub in the aldehyde-induced selective degeneration of SNpc neurons; whether one should consider the enzyme as a victim or a culprit in PD-related pathology is still speculative. Considerable evidence demonstrates that DOPAL accumulation in $\mathrm{SNpc}$ dopaminergic neurons is a natural consequence of ALDH absence or inhibition. Additional factors were described, like increased vulnerability to other aldehydesand neurotoxins-mediated cytotoxicity, i.e. 4-HNE, $\mathrm{MPP}^{+}$ and rotenone, as well as altered modulation of nigrostriatal circuits due to reduced GABA synthesis and release $[33,107,128,135]$. Of interest is the peculiar distribution of ALDH expression in human SNpc dopaminergic neurons where, except for ALDH1A1, no other cytoplasmic ALDH or ALR/AR enzymes are particularly enriched [24, 40, 106]. Consequently, the presence or the absence, the activity or the inhibition of ALDHs, concurrently to other pathological mechanisms, may concur to define the onset and progression of the disease.

Concluding, PD results as a multi-factorial pathology, whose implicated pathways carry additional offshoots themselves. Giving such a level of complexity, any therapeutic approach should be revised to target multiple factors at the time, thus enhancing the probability to succeed. 


\section{Abbreviations}

AEP: Asparagine endopeptidase; ALDH: Aldehyde dehydrogenase: ALR: Aldehyde reductase; APBA: Aminophenylboronic acid; AR: Aldose reductase; BSA: Bovine serum albumin; COX-2: Cyclooxygenase 2; CSF: Cerebrospinal fluid; DA: Dopamine; DAQs: Dopamine quinones; DAT: Dopamine transporter; DOPAC: 3,4-dihydroxyphenylacetic acid; DOPAL: 3,4-dihydroxyphenylacetaldehyde; DOPET:

3,4-dihydroxyphenylethanol; GAPDH: Glyceraldehyde-3-phosphate dehydrogenase; GPX1: Glutathione peroxidase 1; HNE: Hydroxynonenal: HPLC-ECD: High-pressure liquid chromatography - electro-chemical detection; LBs: Lewy Bodies; LDH: Lactate dehydrogenase; MAO: Monoamine oxidase; MDA: Malondialdehyde; MeHg: Methylmercure; mPTP: Mitochondrial permeability transition pore; MSA: Multiple System Atrophy; NBT: Nitroblue tetrazolium; nIRF: Near Infrared Fluorescence; Nurr1: Nuclear receptor-related 1; PD: Parkinson's Disease; Pitx3: Paired-like homeodomain 3; RA: Retinoic acid; ROS: Radical oxygen species; SNpc: Substantia Nigra pars compacta; SOD: Superoxide dismutase; SUMO: Small ubiquitin-like modifier; Syn-TKO: Synuclein triple knock-out; T2DM: Type 2 Diabetes Mellitus; TH: Tyrosine hydroxylase; VMAT-2: Vesicular monoamine transporter type 2; VTA: Ventral-tegmental area; aSyn: aSynuclein; aSyn-OVX: aSyn overexpressing

\section{Authors' contributions}

AM and LB conceived and wrote the article. NP and DB provided critical feedback and helped revising the paper. All authors read and approved the final manuscript.

\section{Funding}

LB was supported by Research Projects of National Interest (PRIN) 2015 prot. 2015T778JW held by the Italian Ministry of Education, University and Research (MIUR). AM was sponsored by Fondazione Ing. Aldo Gini of the University of Padova (2017). NP was supported by a Fondazione Umberto Veronesi post-doctoral fellowship (2018). DB is supported by the Branfman Family Foundation and NIH grant R01-GM086197.

\section{Availability of data and materials}

This is a review article. All data and materials are available.

\section{Ethics approval and consent to participate}

Not applicable.

\section{Consent for publication}

Not applicable.

\section{Competing interests}

The authors declare that they have no competing interests.

\section{Author details}

${ }^{1}$ Department of Biology, University of Padova, Padova, Italy. ${ }^{2}$ Department of Neurosciences, and National Center for Microscopy and Imaging Research, University of California San Diego, La Jolla, CA, USA.

\section{Received: 13 March 2019 Accepted: 22 July 2019}

\section{Published online: 20 August 2019}

\section{References}

1. Dawson TM, Dawson VL. Neuroprotective and neurorestorative strategies for Parkinson's disease. Nat Neurosci. 2002;5:1058-61.

2. Tysnes OB, Storstein A. Epidemiology of Parkinson's disease. J Neural Transm. 2017;124:901-5.

3. Spillantini MG, Schmidt ML, Lee VM-Y, Trojanowski JQ, Jakes R, Goedert M. Alpha-synuclein in Lewy bodies. Nature. 1997;388:839-40.

4. Zarow C, Lyness SA, Mortimer JA, Chui HC. Neuronal loss is greater in the locus Coeruleus than nucleus basalis and substantia Nigra in Alzheimer and Parkinson diseases. Arch Neurol. 2003;60:337-41.

5. Sulzer D, Surmeier DJ. Neuronal vulnerability, pathogenesis, and Parkinson's disease. Mov Disord. 2013;28:41-50.

6. Engelender S, Isacson O. The threshold theory for Parkinson's disease. Trends Neurosci. 2017;40:4-14.

7. Brichta L, Greengard P. Molecular determinants of selective dopaminergic vulnerability in Parkinson's disease: an update. Front Neuroanat. 2014;8:152.
8. Schneider SA, Alcalay RN. Neuropathology of genetic synucleinopathies with parkinsonism: review of the literature. Mov Disord. 2017;32:1504-23.

9. Verstraeten A, Theuns J, Van Broeckhoven C. Progress in unraveling the genetic etiology of Parkinson disease in a genomic era. Trends Genet. 2015;31: 140-9.

10. Sulzer D. Multiple hit hypotheses for dopamine neuron loss in Parkinson's disease. Trends Neurosci. 2007;30:244-50.

11. Carvey PM, Punati A, Newman MB. Progressive dopamine neuron loss in Parkinson's disease: the multiple hit hypothesis. Cell Transplant. 2006;15: 239-50.

12. Surmeier DJ, Obeso JA, Halliday GM. Selective neuronal vulnerability in Parkinson disease. Nat Rev Neurosci. 2017;18:101-13.

13. Surmeier DJ, Obeso JA, Halliday GM. Parkinson's disease is not simply a prion disorder. J Neurosci. 2017;37:9799-807.

14. Chen L, Ding Y, Cagniard B, Van Laar AD, Mortimer A, Chi W, et al. Unregulated cytosolic dopamine causes neurodegeneration associated with oxidative stress in mice. J Neurosci. 2008;28:425-33.

15. Bisaglia M, Filograna R, Beltramini M, Bubacco L. Are dopamine derivatives implicated in the pathogenesis of Parkinson's disease? Ageing Res Rev. 2014;13:107-14.

16. Bisaglia M, Greggio E, Beltramini M, Bubacco L. Dysfunction of dopamine homeostasis: clues in the hunt for novel Parkinson's disease therapies. FASEB J. 2013:27:2101-10.

17. Bisaglia M, Soriano ME, Arduini I, Mammi S, Bubacco L. Molecular characterization of dopamine-derived quinones reactivity toward NADH and glutathione: implications for mitochondrial dysfunction in Parkinson disease. Biochim Biophys Acta. 1802;2010:699-706.

18. Burbulla LF, Song P, Mazzulli JR, Zampese E, Wong YC, Jeon S, et al. Dopamine oxidation mediates mitochondrial and lysosomal dysfunction in Parkinson's disease. Science. 2017;351:1255-61.

19. Longo F, Mercatelli D, Novello S, Arcuri L, Brugnoli A, Vincenzi F, et al. Age-dependent dopamine transporter dysfunction and Serine129 phospho-a-synuclein overload in G2019S LRRK2 mice. Acta Neuropathol Commun. 2017;5:22

20. Mor DE, Tsika E, Mazzulli JR, Gould NS, Kim H, Daniels MJ, et al. Dopamine induces soluble a-synuclein oligomers and nigrostriatal degeneration. Nat Neurosci. 2017;20:1560-8.

21. Roy S. Synuclein and dopamine: the Bonnie and Clyde of Parkinson's disease. Nat Neurosci. 2017;20:1514-5.

22. Goldstein DS, Kopin IJ, Sharabi Y. Catecholamine autotoxicity. Implications for pharmacology and therapeutics of Parkinson disease and related disorders. Pharmacol Ther. 2014;144:268-82.

23. Mattammal MB, Haring JH, Chung HD, Raghu G, Strong R. An endogenous dopaminergic neurotoxin: implication for Parkinson's disease. Neurodegeneration. 1995;4:271-81.

24. Marchitti SA, Deitrich RA, Vasiliou V. Neurotoxicity and metabolism of the catecholamine-derived 3,4-dihydroxyphenylacetaldehyde and 3, 4-dihydroxyphenylglycolaldehyde: the role of aldehyde dehydrogenase. Pharmacol Rev. 2007:59:125-50.

25. Burke WJ, Li SW, Williams EA, Nonneman R, Zahm DS. 3, 4-Dihydroxyphenylacetaldehyde is the toxic dopamine metabolite in vivo: implications for Parkinson's disease pathogenesis. Brain Res. 2003; 989:205-13.

26. Goldstein DS, Sullivan P, Holmes C, Kopin IJ, Basile MJ, Mash DC. Catechols in postmortem brain of patients with Parkinson disease. Eur J Neurol. 2011:18:703-10.

27. Goldstein DS, Sullivan P, Holmes C, Miller GW, Alter S, Strong R, et al. Determinants of buildup of the toxic dopamine metabolite DOPAL in Parkinson's disease. J Neurochem. 2013;126:591-603.

28. Wey MC-Y, Fernandez E, Martinez PA, Sullivan P, Goldstein DS, Strong R. Neurodegeneration and motor dysfunction in mice lacking cytosolic and mitochondrial aldehyde dehydrogenases: implications for Parkinson's disease. PLoS One. 2012;7:e31522.

29. Filograna R, Civiero L, Ferrari V, Codolo G, Greggio E, Bubacco L, et al. Analysis of the Catecholaminergic phenotype in human SH-SY5Y and BE (2)-M17 neuroblastoma cell lines upon differentiation. PLoS One. 2015;10:e0136769.

30. Goldstein DS, Holmes C, Kopin IJ, Sharabi Y. Intra-neuronal vesicular uptake of catecholamines is decreased in patients with Lewy body diseases. J Clin Invest. 2011;121:3320-30.

31. Goldstein DS, Holmes C, Sullivan P, Jinsmaa Y, Kopin IJ, Sharabi Y. Elevated cerebrospinal fluid ratios of cysteinyl-dopamine/3,4-dihydroxyphenylacetic acid in parkinsonian synucleinopathies. Parkinsonism Relat Disord. 2016;31:79-86. 
32. Panneton WM, Kumar VB, Gan Q, Burke WJ, Galvin JE. The neurotoxicity of DOPAL: behavioral and stereological evidence for its role in Parkinson disease pathogenesis. PLoS One. 2010;5:e15251.

33. Lamensdorf I, Eisenhofer G, Harvey-White J, Nechustan A, Kirk K, Kopin IJ. 3,4-Dihydroxyphenylacetaldehyde potentiates the toxic effects of metabolic stress in PC12 cells. Brain Res. 2000:868:191-201.

34. Rees JN, Florang VR, Anderson DG, Doorn JA. Lipid peroxidation products inhibit dopamine catabolism yielding aberrant levels of a reactive intermediate. Chem Res Toxicol. 2007;20:1536-42.

35. Li SW, Lin TS, Minteer S, Burke WJ. 3,4-Dihydroxyphenylacetaldehyde and hydrogen peroxide generate a hydroxyl radical: possible role in Parkinson's disease pathogenesis. Brain Res Mol Brain Res. 2001;93:1-7.

36. Rees JN, Florang VR, Eckert LL, Doorn JA. Protein reactivity of 3,4Dihydroxyphenylacetaldehyde, a toxic dopamine metabolite, is dependent on both the aldehyde and the catechol. Chem Res Toxicol. 2009;22:1256-63.

37. Anderson DG, Florang VR, Schamp JH, Buettner GR, Doorn JA. Antioxidant -mediated modulation of protein reactivity for 3,4-

Dihydroxyphenylacetaldehyde, a toxic dopamine metabolite. Chem Res Toxicol. 2016;29:1098-107.

38. Follmer C, Coelho-Cerqueira E, Yatabe-Franco DY, Araujo GDT, Pinheiro AS, Domont GB, et al. Oligomerization and membrane-binding properties of covalent adducts formed by the interaction of a-Synuclein with the toxic dopamine metabolite 3,4-Dihydroxyphenylacetaldehyde (DOPAL). J Biol Chem. 2015;290:27660-79.

39. Anderson DG, Mariappan SVS, Buettner GR, Doorn JA. Oxidation of 3 , 4-Dihydroxyphenylacetaldehyde, a toxic dopaminergic metabolite, to a Semiquinone radical and an ortho -Quinone. J Biol Chem. 2011;286: 26978-86.

40. Liu G, Yu J, Ding J, Xie C, Sun L, Rudenko I, et al. Aldehyde dehydrogenase 1 defines and protects a nigrostriatal dopaminergic neuron subpopulation. J Clin Invest. 2014;124:3032-46.

41. Plotegher N, Berti G, Ferrari E, Tessari I, Zanetti M, Lunelli L, et al. DOPAL derived alpha-synuclein oligomers impair synaptic vesicles physiological function. Sci Rep. 2017;7:40699.

42. Jinsmaa Y, Sharabi Y, Sullivan P, Isonaka R, Goldstein DS. 3,4Dihydroxyphenylacetaldehyde-induced protein modifications and their mitigation by N-acetylcysteine. J Pharmacol Exp Ther. 2018;366:113-24.

43. Mazzulli JR, Burbulla LF, Krainc D, Ischiropoulos $H$. Detection of free and protein-bound ortho -Quinones by near-infrared fluorescence. Anal Chem. 2016;88:2399-405

44. Burke WJ, Kumar VB, Pandey N, Panneton WM, Gan Q, Franko MW, et al. Aggregation of alpha-synuclein by DOPAL, the monoamine oxidase metabolite of dopamine. Acta Neuropathol. 2008;115:193-203.

45. Plotegher N, Bubacco L. Lysines, Achilles' heel in alpha-synuclein conversion to a deadly neuronal endotoxin. Ageing Res Rev. 2016;26:62-71.

46. Vermeer LMM, Florang VR, Doorn JA. Catechol and aldehyde moieties of 3,4-dihydroxyphenylacetaldehyde contribute to tyrosine hydroxylase inhibition and neurotoxicity. Brain Res. 2012;1474:100-9.

47. Vanle BC, Florang VR, Murry DJ, Aguirre AL, Doorn JA. Inactivation of glyceraldehyde-3-phosphate dehydrogenase by the dopamine metabolite, 3,4-dihydroxyphenylacetaldehyde. Biochem Biophys Res Commun. 2017;492: 275-81.

48. LaVoie MJ, Ostaszewski BL, Weihofen A, Schlossmacher MG, Selkoe DJ. Dopamine covalently modifies and functionally inactivates parkin. Nat Med. 2005;11:1214-21.

49. Kristal BS, Conway AD, Brown AM, Jain JC, Ulluci PA, Li SW, et al. Selective dopaminergic vulnerability: 3,4-dihydroxyphenylacetaldehyde targets mitochondria. Free Radic Biol Med. 2001;30:924-31.

50. Van Laar VS, Mishizen AJ, Cascio M, Hastings TG. Proteomic identification of dopamine-conjugated proteins from isolated rat brain mitochondria and SH-SY5Y cells. Neurobiol Dis. 2009;34:487-500.

51. Biosa A, Arduini I, Soriano ME, Giorgio V, Bernardi P, Bisaglia M, et al. Dopamine oxidation products as mitochondrial endotoxins, a potential molecular mechanism for preferential neurodegeneration in Parkinson's disease. ACS Chem Neurosci. 2018;9:2849-58.

52. Wilkinson KA, Henley JM. Mechanisms, regulation and consequences of protein SUMOylation. Biochem J. 2010;428:133-45.

53. Martin S, Nishimune A, Mellor JR, Henley JM. SUMOylation regulates kainate -receptor-mediated synaptic transmission. Nature. 2007;447:321-5.

54. Hayashi T, Seki M, Maeda D, Wang W, Kawabe Y, Seki T, et al. Ubc9 is essential for viability of higher eukaryotic cells. Exp Cell Res. 2002;280:212-21.
55. Kunadt M, Eckermann K, Stuendl A, Gong J, Russo B, Strauss K, et al Extracellular vesicle sorting of a-Synuclein is regulated by sumoylation. Acta Neuropathol. 2015;129:695-713.

56. Abeywardana T, Pratt MR. Extent of inhibition of a-synuclein aggregation in vitro by SUMOylation is conjugation site- and SUMO isoform-selective. Biochemistry. 2015;54:959-61.

57. Shahpasandzadeh H, Popova B, Kleinknecht A, Fraser PE, Outeiro TF, Braus $\mathrm{GH}$. Interplay between sumoylation and phosphorylation for protection against a-synuclein inclusions. J Biol Chem. 2014;289:31224-40.

58. Nakayasu ES, Burnet MC, Walukiewicz HE, Wilkins CS, Shukla AK, Brooks S, et al. Ancient regulatory role of lysine acetylation in central metabolism. MBio. 2017;8:e01894-17.

59. Chaibva M, Jawahery S, Pilkington AW, Arndt JR, Sarver O, Valentine S, et al. Acetylation within the first 17 residues of huntingtin exon 1 alters aggregation and lipid binding. Biophys J. 2016;111:349-62.

60. Jeong H, Then F, Melia TJ, Mazzulli JR, Cui L, Savas JN, et al. Acetylation targets mutant huntingtin to autophagosomes for degradation. Cell. 2009;137:60-72.

61. Lee JH, Ying J, Bax A. Nuclear magnetic resonance observation of aSynuclein membrane interaction by monitoring the acetylation reactivity of its lysine side chains. Biochemistry. 2016;55:4949-59.

62. Mexas LM, Florang VR, Doorn JA. Inhibition and covalent modification of tyrosine hydroxylase by 3,4-dihydroxyphenylacetaldehyde, a toxic dopamine metabolite. Neurotoxicology. 2011;32:471-7.

63. De Lazzari F, Bubacco L, Whitworth AJ, Bisaglia M. Superoxide radical Dismutation as new therapeutic strategy in Parkinson's disease. Aging Dis. 2017;9:716-28.

64. Werner-Allen JW, Levine RL, Bax A. Superoxide is the critical driver of DOPAL autoxidation, lysyl adduct formation, and crosslinking of a-synuclein Biochem Biophys Res Commun. 2017;487:281-6.

65. Winner B, Jappelli R, Maji SK, Desplats PA, Boyer L, Aigner S, et al. In vivo demonstration that alpha-synuclein oligomers are toxic. Proc Natl Acad Sci U S A. 2011;108:4194-9.

66. Wong YC, Krainc D. a-Synuclein toxicity in neurodegeneration: mechanism and therapeutic strategies. Nat Med. 2017;23:1-13.

67. Fusco G, Sanz-Hernandez M, De Simone A. Order and disorder in the physiological membrane binding of a-synuclein. Curr Opin Struct Biol. 2018:48:49-57.

68. Iwai A, Masliah E, Yoshimoto M, Ge N, Flanagan $L$, de Silva HA, et al. The precursor protein of non-a beta component of Alzheimer's disease amyloid is a presynaptic protein of the central nervous system. Neuron. 1995;14:467-75.

69. Wilhelm BG, Mandad S, Truckenbrodt S, Krohnert K, Schafer C, Rammner B, et al. Composition of isolated synaptic boutons reveals the amounts of vesicle trafficking proteins. Science. 2014;344:1023-8.

70. Burré J. The synaptic function of a-Synuclein. J Park Dis. 2015;5:699-713.

71. Meiser J, Weindl D, Hiller K. Complexity of dopamine metabolism. Cell Commun Signal. 2013;11:34.

72. Goldstein DS, Sullivan P, Cooney A, Jinsmaa Y, Sullivan R, Gross DJ, et al Vesicular uptake blockade generates the toxic dopamine metabolite 3, 4-dihydroxyphenylacetaldehyde in PC12 cells: relevance to the pathogenesis of Parkinson's disease. J Neurochem. 2012;123:932-43.

73. Dexter DT, Wells FR, Lees AJ, Agid F, Agid Y, Jenner P, et al. Increased nigral iron content and alterations in other metal ions occurring in brain in Parkinson's disease. J Neurochem. 1989:52:1830-6.

74. Jinsmaa Y, Sullivan P, Gross D, Cooney A, Sharabi Y, Goldstein DS. Divalent metal ions enhance DOPAL-induced oligomerization of alpha-synuclein. Neurosci Lett. 2014;569:27-32.

75. Lima V d A, do Nascimento LA, Eliezer D, Follmer C. Role of Parkinson's Disease-linked Mutations and N-Terminal Acetylation on the Oligomerization of a-Synuclein Induced by DOPAL. ACS Chem Neurosci. 2018;10:690-703.

76. Kang SS, Ahn EH, Zhang Z, Liu X, Manfredsson FP, Sandoval IM, et al. aSynuclein stimulation of monoamine oxidase- $B$ and legumain protease mediates the pathology of Parkinson's disease. EMBO J. 2018;37:e98878.

77. Janezic S, Threlfell S, Dodson PD, Dowie MJ, Taylor TN, Potgieter D, et al. Deficits in dopaminergic transmission precede neuron loss and dysfunction in a new Parkinson model. Proc Natl Acad Sci. 2013;110:E4016-25.

78. Dettmer U, Newman AJ, von Saucken VE, Bartels T, Selkoe D. KTKEGV repeat motifs are key mediators of normal a-synuclein tetramerization: their mutation causes excess monomers and neurotoxicity. Proc Natl Acad Sci U S A. 2015;112:9596-601.

79. Logan T, Bendor J, Toupin C, Thorn K, Edwards RH. a-Synuclein promotes dilation of the exocytotic fusion pore. Nat Neurosci. 2017;20:681-9. 
80. Zhang Z, Su Kang S, Liu X, Hee Ahn E, Zhang Z, He L, et al. Asparagine endopeptidase cleaves a-synuclein and mediates pathologic activities in Parkinson's disease. Nat Struct Mol Biol. 2017;24:632-42.

81. Burre J, Sharma M, Tsetsenis T, Buchman V, Etherton MR, Sudhof TC. a-Synuclein promotes SNARE-complex assembly in vivo and in vitro. Science. 2010;329:1663-7.

82. Nemani VM, Lu W, Berge V, Nakamura K, Onoa B, Lee MK, et al. Increased expression of a-Synuclein reduces neurotransmitter release by inhibiting synaptic vesicle Reclustering after endocytosis. Neuron. 2010;65:66-79.

83. Senior SL, Ninkina N, Deacon R, Bannerman D, Buchman VL, Cragg SJ, et al. Increased striatal dopamine release and hyperdopaminergic-like behaviour in mice lacking both alpha-synuclein and gamma-synuclein. Eur J Neurosci. 2008;27:947-57.

84. Anwar S, Peters O, Millership S, Ninkina N, Doig N, Connor-Robson N, et al. Functional alterations to the nigrostriatal system in mice lacking all three members of the Synuclein family. J Neurosci. 2011;31:7264-74.

85. Masato A, Berti G, Plotegher N, De Lazzari F, Bisaglia M, Greggio E, Boassa D, Bubacco L. Altered dopamine metabolism leads to a unique impaired aSynuclein proteostasis in Parkinson's Disease. 750.18. 2018 Neuroscience Meeting Planner. San Diego: Society for Neuroscience; 2018. Online.

86. Jinsmaa $Y$, Sullivan $P$, Sharabi $Y$, Goldstein DS. DOPAL is transmissible to and oligomerizes alpha-synuclein in human glial cells. Auton Neurosci. 2016;194:46-51.

87. Goldstein DS, Sullivan P, Holmes C, Kopin IJ, Sharabi Y, Mash DC. Decreased vesicular storage and aldehyde dehydrogenase activity in multiple system atrophy. Parkinsonism Relat Disord. 2015;21:567-72.

88. Pifl C, Rajput A, Reither H, Blesa J, Cavada C, Obeso JA, et al. Is Parkinson's disease a vesicular dopamine storage disorder? Evidence from a study in isolated synaptic vesicles of human and nonhuman primate striatum. J Neurosci. 2014;34:8210-8

89. Caudle WM, Richardson JR, Wang MZ, Taylor TN, Guillot TS, McCormack AL, et al. Reduced vesicular storage of dopamine causes progressive nigrostriatal neurodegeneration. J Neurosci. 2007;27:8138-48.

90. Santos JR, Cunha JAS, Dierschnabel AL, Campêlo CLC, Leão AHFF, Silva $A F$, et al. Cognitive, motor and tyrosine hydroxylase temporal impairment in a model of parkinsonism induced by reserpine. Behav Brain Res. 2013;253:68-77.

91. Fernandez E, Koek W, Ran Q, Gerhardt GA, France CP, Strong R. Monoamine metabolism and behavioral responses to ethanol in mitochondrial aldehyde dehydrogenase knockout mice. Alcohol Clin Exp Res. 2006;30:1650-8.

92. Leão AHFF, Sarmento-Silva AJ, Santos JR, Ribeiro AM, Silva RH. Molecular, neurochemical, and behavioral hallmarks of reserpine as a model for Parkinson's disease: new perspectives to a long-standing model. Brain Pathol. 2015;25:377-90.

93. Brighina L, Riva C, Bertola F, Saracchi E, Fermi S, Goldwurm S, et al. Analysis of vesicular monoamine transporter 2 polymorphisms in Parkinson's disease. Neurobiol Aging. 2013;34:1712.e9-13.

94. Nguyen M, Krainc D. LRRK2 phosphorylation of auxilin mediates synaptic defects in dopaminergic neurons from patients with Parkinson's disease. Proc Natl Acad Sci U S A. 2018;115:5576-81.

95. Agid Y, Javoy F, Youdim MB. Monoamine oxidase and aldehyde dehydrogenase activity in the striatum of rats after 6-hydroxydopamine lesion of the nigrostriatal pathway. Br J Pharmacol. 1973;48:175-8.

96. Chamoli M, Chinta SJ, Andersen JK. An inducible MAO-B mouse model of Parkinson's disease: a tool towards better understanding basic disease mechanisms and developing novel therapeutics. J Neural Transm. 2018;125:1651-8.

97. Camell CD, Sander J, Spadaro O, Lee A, Nguyen KY, Wing A, et al. Inflammasome-driven catecholamine catabolism in macrophages blunts lipolysis during ageing. Nature. 2017;550:119.

98. Nicotra A, Pierucci F, Parvez H, Senatori O. Monoamine oxidase expression during development and aging. Neurotoxicology. 2004;25:155-65.

99. Damier P, Kastner A, Agid Y, Hirsch EC. Does monoamine oxidase type B play a role in dopaminergic nerve cell death in Parkinson's disease? Neurology. 1996;46:1262-9.

100. Sampaio TF, dos Santos EUD, de Lima GDC, dos Anjos RSG, da Silva RC, Asano AGC, et al. MAO-B and COMT genetic variations associated with levodopa treatment response in patients with Parkinson's disease. J Clin Pharmacol. 2018;58:920-6.

101. Sun $Y-X$, Wang $X-H, X u A-H$, Zhao J-H. Functional polymorphisms of the MAO gene with Parkinson disease susceptibility: a meta-analysis. J Neurol Sci. 2014;345:97-105.
102. Kurth JH, Kurth MC, Poduslo SE, Schwankhaus JD. Association of a monoamine oxidase B allele with Parkinson's disease. Ann Neurol. 1993; 33:368-72.

103. Mallajosyula JK, Kaur D, Chinta SJ, Rajagopalan S, Rane A, Nicholls DG, et al. MAO-B elevation in mouse brain astrocytes results in Parkinson's pathology. PLoS One. 2008;3:e1616.

104. Marchitti SA, Brocker C, Stagos D, Vasiliou V. Non-P450 aldehyde oxidizing enzymes: the aldehyde dehydrogenase superfamily. Expert Opin Drug Metab Toxicol. 2008;4:697-720.

105. McCaffery $P$, Dräger UC. High levels of a retinoic acid-generating dehydrogenase in the meso-telencephalic dopamine system. Proc Natl Acad Sci U S A. 1994;91:7772-6.

106. Cai H, Liu G, Sun L, Ding J. Aldehyde dehydrogenase 1 making molecular inroads into the differential vulnerability of nigrostriatal dopaminergic neuron subtypes in Parkinson's disease. Transl Neurodegener. 2014;3:27.

107. Kim J-I, Ganesan S, Luo SX, Wu Y-W, Park E, Huang EJ, et al. Aldehyde dehydrogenase 1a1 mediates a GABA synthesis pathway in midbrain dopaminergic neurons. Science. 2015;350:102-6.

108. Jacobs FMJ, Smits SM, Noorlander CW, von Oerthel L, van der Linden AJA, Burbach JPH, et al. Retinoic acid counteracts developmental defects in the substantia nigra caused by Pitx3 deficiency. Development. 2007;134:2673-84.

109. Sgobio C, Wu J, Zheng W, Chen X, Pan J, Salinas AG, et al. Aldehyde dehydrogenase 1-positive nigrostriatal dopaminergic fibers exhibit distinct projection pattern and dopamine release dynamics at mouse dorsal striatum. Sci Rep. 2017;7:5283.

110. Tritsch NX, Ding JB, Sabatini BL. Dopaminergic neurons inhibit striatal output through non-canonical release of GABA. Nature. 2012;490:262-6.

111. Granger AJ, Wallace ML, Sabatini BL. Multi-transmitter neurons in the mammalian central nervous system. Curr Opin Neurobiol. 2017;45:85-91.

112. Ambroziak W, Pietruszko R. Human aldehyde dehydrogenase: metabolism of putrescine and histamine. Alcohol Clin Exp Res. 1987;11:528-32.

113. Mackerell AD, Blatter EE, Pietruszko R. Human aldehyde dehydrogenase: kinetic identification of the isozyme for which biogenic aldehydes and acetaldehyde compete. Alcohol Clin Exp Res. 1986;10:266-70.

114. Ambroziak W, Pietruszko R. Human aldehyde dehydrogenase. Activity with aldehyde metabolites of monoamines, diamines, and polyamines. J Biol Chem. 1991;266:13011-8.

115. Galter D, Buervenich S, Carmine A, Anvret M, Olson L. ALDH1 mRNA: presence in human dopamine neurons and decreases in substantia nigra in Parkinson's disease and in the ventral tegmental area in schizophrenia. Neurobiol Dis. 2003;14:637-47.

116. Yoshida A, Hsu LC, Davé V. Retinal oxidation activity and biological role of human cytosolic aldehyde dehydrogenase. Enzyme. 1992;46:239-44.

117. Xiao T, Shoeb M, Siddiqui MS, Zhang M, Ramana KV, Srivastava SK, et al. Molecular cloning and oxidative modification of human lens ALDH1A1: implication in impaired detoxification of lipid aldehydes. J Toxicol Environ Health A. 2009;72:577-84.

118. King G, Holmes R. Human corneal and lens aldehyde dehydrogenases. Purification and properties of human lens ALDH1 and differential expression as major soluble proteins in human lens (ALDH1) and cornea (ALDH3). Adv Exp Med Biol. 1997:414:19-27.

119. Manzer R, Qamar L, Estey T, Pappa A, Petersen DR, Vasiliou V. Molecular cloning and baculovirus expression of the rabbit corneal aldehyde dehydrogenase (ALDH1A1) cDNA. DNA Cell Biol. 2003;22:329-38.

120. Klyosov AA, Rashkovetsky LG, Tahir MK, Keung WM. Possible role of liver cytosolic and mitochondrial aldehyde dehydrogenases in acetaldehyde metabolism. Biochemistry. 1996;35:4445-56.

121. Mackerell $A D$, Pietruszko R. Chemical modification of human aldehyde dehydrogenase by physiological substrate. Biochim Biophys Acta. 1987;911:306-17.

122. Mitchell DY, Petersen DR. The oxidation of alpha-beta unsaturated aldehydic products of lipid peroxidation by rat liver aldehyde dehydrogenases. Toxicol Appl Pharmacol. 1987;87:403-10

123. Vasiliou V, Pappa A, Estey T. Role of human aldehyde dehydrogenases in endobiotic and xenobiotic metabolism. Drug Metab Rev. 2004;36: 279-99.

124. Reichard JF, Vasiliou V, Petersen DR. Characterization of 4-hydroxy-2-nonenal metabolism in stellate cell lines derived from normal and cirrhotic rat liver. Biochim Biophys Acta. 2000;1487:222-32.

125. Fitzmaurice AG, Rhodes SL, Cockburn M, Ritz B, Bronstein JM. Aldehyde dehydrogenase variation enhances effect of pesticides associated with Parkinson disease. Neurology. 2014;82:419-26. 
126. Zhang X, Ye Y-L, Wang Y-N, Liu F-F, Liu X-X, Hu B-L, et al. Aldehyde dehydrogenase 2 genetic variations may increase susceptibility to Parkinson's disease in Han Chinese population. Neurobiol Aging. 2015;36:2660.e9-13.

127. Zhao CC, Cai HB, Wang H, Pan SY. Role of ADH2 and ALDH2 gene polymorphisms in the development of Parkinson's disease in a Chinese population. Genet Mol Res. 2016;15:gmr15038606.

128. Grünblatt E, Riederer P. Aldehyde dehydrogenase (ALDH) in Alzheimer's and Parkinson's disease. J Neural Transm. 2016;123:83-90.

129. Lin X, Parisiadou L, Sgobio C, Liu G, Yu J, Sun L, et al. Conditional expression of Parkinson's disease-related mutant -Synuclein in the midbrain dopaminergic neurons causes progressive neurodegeneration and degradation of transcription factor nuclear receptor related 1. J Neurosci. 2012;32:9248-64.

130. Mandel SA, Fishman T, Youdim MBH. Gene and protein signatures in sporadic Parkinson's disease and a novel genetic model of PD. Parkinsonism Relat Disord. 2007;13:S242-7.

131. Werner CJ, Heyny-von Haussen R, Mall G, Wolf S. Proteome analysis of human substantia nigra in Parkinson's disease. Proteome Sci. 2008;6:8.

132. Liu G, Sgobio C, Gu X, Sun L, Lin X, Yu J, et al. Selective expression of Parkinson's disease-related leucine-rich repeat kinase 2 G2019S missense mutation in midbrain dopaminergic neurons impairs dopamine release and dopaminergic gene expression. Hum Mol Genet. 2015;24:5299-312.

133. Corrigan FM, Murray L, Wyatt CL, Shore RF. Diorthosubstituted polychlorinated biphenyls in caudate nucleus in Parkinson's disease. Exp Neurol. 1998;150:339-42.

134. Fitzmaurice AG, Rhodes SL, Lulla A, Murphy NP, Lam HA, O'Donnell KC, et al. Aldehyde dehydrogenase inhibition as a pathogenic mechanism in Parkinson disease. Proc Natl Acad Sci U S A. 2013;110:636-41.

135. Jinsmaa Y, Florang VR, Rees JN, Anderson DG, Strack S, Doorn JA. Products of oxidative stress inhibit aldehyde oxidation and reduction pathways in dopamine catabolism yielding elevated levels of a reactive intermediate. Chem Res Toxicol. 2009:22:835-41.

136. Florang VR, Rees JN, Brogden NK, Anderson DG, Hurley TD, Doorn JA. Inhibition of the oxidative metabolism of 3,4-dihydroxyphenylacetaldehyde, a reactive intermediate of dopamine metabolism, by 4-hydroxy-2-nonenal. Neurotoxicology. 2007:28:76-82.

137. Legros H, Dingeval M-G, Janin F, Costentin J, Bonnet J-J. Toxicity of a treatment associating dopamine and disulfiram for Catecholaminergic neuroblastoma SH-SY5Y cells: relationships with 3,4Dihydroxyphenylacetaldehyde formation. Neurotoxicology. 2004;25:365-75.

138. Rooke N, Li DJ, Li J, Keung WM. The mitochondrial monoamine oxidase-aldehyde dehydrogenase pathway: a potential site of action of daidzin. J Med Chem. 2000; 43:4169-79.

139. Doorn JA, Florang VR, Schamp JH, Vanle BC. Aldehyde dehydrogenase inhibition generates a reactive dopamine metabolite autotoxic to dopamine neurons. Parkinsonism Relat Disord. 2014;20(Suppl 1):S73-5.

140. Bai X, Wey MC-Y, Martinez PA, Shi C, Fernandez E, Strong R. Neurochemical and motor changes in mice with combined mutations linked to Parkinson's disease. Pathobiol Aging Age Relat Dis. 2017;7:1267855.

141. Casida JE, Ford B, Jinsmaa Y, Sullivan P, Cooney A, Goldstein DS. Benomyl, aldehyde dehydrogenase, DOPAL, and the catecholaldehyde hypothesis for the pathogenesis of Parkinson's disease. Chem Res Toxicol. 2014;27:1359-61.

142. Lulla A, Barnhill L, Bitan G, Ivanova MI, Nguyen B, O'Donnell K, et al. Neurotoxicity of the Parkinson disease-associated pesticide Ziram is Synuclein-dependent in zebrafish embryos. Environ Health Perspect. 2016; 124:1766-75.

143. Grunblatt E, Mandel S, Jacob-Hirsch J, Zeligson S, Amariglo N, Rechavi G, et al. Gene expression profiling of parkinsonian substantia nigra pars compacta; alterations in ubiquitin-proteasome, heat shock protein, iron and oxidative stress regulated proteins, cell adhesion/cellular matrix and vesicle trafficking genes. J Neural Transm. 2004;111:1543-73.

144. Michel TM, Käsbauer L, Gsell W, Jecel J, Sheldrick AJ, Cortese M, et al. Aldehyde dehydrogenase 2 in sporadic Parkinson's disease. Parkinsonism Relat Disord. 2014;20:568-72.

145. Yoritaka A, Hattori N, Uchida K, Tanaka M, Stadtman ER, Mizuno Y. Immunohistochemical detection of 4-hydroxynonenal protein adducts in Parkinson disease. Proc Natl Acad Sci U S A. 1996;93:2696-701.

146. Koppaka V, Thompson DC, Chen Y, Ellermann M, Nicolaou KC, Juvonen RO, et al. Aldehyde dehydrogenase inhibitors: a comprehensive review of the pharmacology, mechanism of action, substrate specificity, and clinical application. Pharmacol Rev. 2012;64:520-39.
147. Kanthasamy AAG, Kitazawa M, Kanthasamy AAG, Anantharam V. Dieldrin -induced neurotoxicity: relevance to Parkinson's disease pathogenesis. Neurotoxicology. 2005;26:701-19.

148. Wang A, Costello S, Cockburn M, Zhang X, Bronstein J, Ritz B. Parkinson's disease risk from ambient exposure to pesticides. Eur J Epidemiol. 2011;26: 547-55.

149. Chou AP, Maidment N, Klintenberg R, Casida JE, Li S, Fitzmaurice AG, et al. Ziram causes dopaminergic cell damage by inhibiting E1 ligase of the proteasome. J Biol Chem. 2008;283:34696-703.

150. Dreiem A, Shan M, Okoniewski RJ, Sanchez-Morrissey S, Seegal RF. Methylmercury inhibits dopaminergic function in rat pup synaptosomes in an age-dependent manner. Neurotoxicol Teratol. 2009;31:312-7.

151. Tiernan $C T$, Edwin EA, Hawong H-Y, Ríos-Cabanillas M, Goudreau $L$, Atchison WD, et al. Methylmercury impairs canonical dopamine metabolism in rat undifferentiated pheochromocytoma (PC12) cells by indirect inhibition of aldehyde dehydrogenase. Toxicol Sci. 2015;144:347-56.

152. Lamensdorf I, Eisenhofer G, Harvey-White J, Hayakawa Y, Kirk K, Kopin IJ. Metabolic stress in PC12 cells induces the formation of the endogenous dopaminergic neurotoxin, 3,4-dihydroxyphenylacetaldehyde. J Neurosci Res. 2000;60:552-8

153. Decressac M, Volakakis N, Björklund A, Perlmann T. NURR1 in Parkinson disease — from pathogenesis to therapeutic potential. Nat Rev Neurol. 2013;9:629-36.

154. Volpicelli F, De Gregorio R, Pulcrano S, Perrone-Capano C, di Porzio U, Bellenchi GC. Direct regulation of Pitx3 expression by Nurr1 in culture and in developing mouse midbrain. PLoS One. 2012;7:e30661.

155. Chu Y, Kompoliti K, Cochran EJ, Mufson EJ, Kordower JH. Age-related decreases in Nurr1 immunoreactivity in the human substantia nigra. J Comp Neurol. 2002:450:203-14

156. Chiu C-C, Yeh T-H, Lai S-C, Wu-Chou Y-H, Chen C-H, Mochly-Rosen D, et al. Neuroprotective effects of aldehyde dehydrogenase 2 activation in rotenone-induced cellular and animal models of parkinsonism. Exp Neurol. 2015:263:244-53.

157. Chen C-H, Budas GR, Churchill EN, Disatnik M-H, Hurley TD, Mochly-Rosen D. Activation of aldehyde dehydrogenase-2 reduces ischemic damage to the heart. Science. 2008;321:1493-5.

158. Perez-Miller S, Younus H, Vanam R, Chen C-H, Mochly-Rosen D, Hurley TD. Alda- 1 is an agonist and chemical chaperone for the common human aldehyde dehydrogenase 2 variant. Nat Struct Mol Biol. 2010;17:159-64.

159. Yu R-L, Tan C-H, Lu Y-C, Wu R-M. Aldehyde dehydrogenase 2 is associated with cognitive functions in patients with Parkinson's disease. Sci Rep. 2016; 6:30424.

160. Madadi F, Khaniani MS, Shandiz EE, Ayromlou H, Najmi S, Emamalizadeh B, et al. Genetic analysis of the ZNF512B, SLC41A1, and ALDH2 polymorphisms in Parkinson's disease in the Iranian population. Genet Test Mol Biomarkers. 2016;20:629-32.

161. Anderson DW, Schray RC, Duester G, Schneider JS. Functional significance of aldehyde dehydrogenase ALDH1A1 to the nigrostriatal dopamine system. Brain Res. 2011;1408:81-7.

162. Duke DC, Moran LB, Pearce RKB, Graeber MB. The medial and lateral substantia nigra in Parkinson's disease: mRNA profiles associated with higher brain tissue vulnerability. Neurogenetics. 2007:8:83-94.

163. Goldstein DS, Jinsmaa Y, Sullivan P, Holmes C, Kopin IJ, Sharabi Y. Comparison of monoamine oxidase inhibitors in decreasing production of the autotoxic dopamine metabolite 3,4-Dihydroxyphenylacetaldehyde in PC12 cells. J Pharmacol Exp Ther. 2016;356:483-92

164. Kumar VB, Hsu F-F, Lakshmi VM, Gillespie KN, Burke WJ. Aldehyde adducts inhibit 3,4-dihydroxyphenylacetaldehyde-induced a-synuclein aggregation and toxicity: implication for Parkinson neuroprotective therapy. Eur J Pharmacol. 2019;845:65-73.

165. Biosa A, Outeiro TF, Bubacco L, Bisaglia M. Diabetes mellitus as a risk factor for Parkinson's disease: a molecular point of view. Mol Neurobiol. 2018;55: 8754-63.

166. Rotermund C, Machetanz G, Fitzgerald JC. The therapeutic potential of metformin in neurodegenerative diseases. Front Endocrinol (Lausanne). 2018;9:400.

167. Oueslati A. Implication of alpha-Synuclein phosphorylation at S129 in Synucleinopathies: what have we learned in the last decade? J Park Dis. 2016;6:39-51.

168. Pérez-Revuelta BI, Hettich MM, Ciociaro A, Rotermund C, Kahle PJ, Krauss S, et al. Metformin lowers Ser-129 phosphorylated a-synuclein levels via mTOR -dependent protein phosphatase 2A activation. Cell Death Dis. 2014;5:e1209. 
169. Katila N, Bhurtel S, Shadfar S, Srivastav S, Neupane S, Ojha U, et al. Metformin lowers a-synuclein phosphorylation and upregulates neurotrophic factor in the MPTP mouse model of Parkinson's disease. Neuropharmacology. 2017;125:396-407.

\section{Publisher's Note}

Springer Nature remains neutral with regard to jurisdictional claims in published maps and institutional affiliations.

Ready to submit your research? Choose BMC and benefit from:

- fast, convenient online submission

- thorough peer review by experienced researchers in your field

- rapid publication on acceptance

- support for research data, including large and complex data types

- gold Open Access which fosters wider collaboration and increased citations

- maximum visibility for your research: over $100 \mathrm{M}$ website views per year

At $B M C$, research is always in progress.

Learn more biomedcentral.com/submissions 